\title{
Cohomology of Power Sets with Applications in Quantum Probability
}

\author{
J. M. Lindsay* and K. R. Parthasarathy \\ Indian Statistical Institute, (Delhi Centre), 7, S.J.S. Sansanwal Marg, New Delhi-110016, India
}

\begin{abstract}
Square integrable Wiener functionals may be represented as sums of multiple Itô integrals. This leads to an identification of such functionals with square integrable functions on the symmetric measure space of the Lebesgue space $R_{+}$. When the pointwise product of Wiener functionals is thus carried over, the product takes a pleasing form (cf. Wick's theorem) and various non-commutative perturbations of this "Wiener product" have been considered. Here we employ cohomological arguments to analyse deformations of an abstract Wiener product. This leads to the construction of Lévy fields which are neither bosonic nor fermionic, and also gives rise to homotopies between quasi-free boson and fermion fields. Finally we unify existence and uniqueness results for quantum stochastic differential equations by treating mixed noise differential equations.
\end{abstract}

\section{Introduction}

Any square integrable Wiener functional $F$ has an expansion in terms of multiple Itô integrals:

$$
F=f_{0}+\sum_{n=1}^{\infty} \int \cdots \int_{\nabla^{n}} f_{n}\left(t_{1}, \ldots, t_{n}\right) \mathrm{dB}_{t_{1}} \cdots \mathrm{dB}_{t_{n}}
$$

where $\nabla^{n}$ is the increasing quadrant $\left\{\mathbf{t} \in \mathbb{R}_{4}^{n}: t_{1}<\cdots<t_{n}\right\}$. The sequence $\left\{f_{n}\right\}$ may be viewed as a single function $f$ on the collection $\Gamma\left(\mathbb{R}_{+}\right)$of all subsets of $\mathbb{R}_{+}$having finite cardinality:

$$
f(\varnothing)=f_{0} ; \quad f(\sigma)=f_{n}(\mathbf{s}) \text { for } \quad n=\# \sigma \geqq 1,
$$

where $s_{1}, s_{2}, \ldots, s_{n}$ is an enumeration of the set $\sigma$ in increasing order. There is a natural measure $\lambda$ on $\Gamma\left(\mathbb{R}_{+}\right)$, derived from Lebesgue measure on $\mathbb{R}_{+}$, for which the correspondence $F \rightarrow f$ is an isometric isomorphism from $\mathscr{W}$, the space of square integrable Wiener functionals, to $\mathscr{F}=L^{2}\left(\Gamma\left(\mathbb{R}_{+}\right), \lambda\right)$. Under pointwise

\footnotetext{
* Address from September 1988; Department of Mathematics, King's College, London WC2R2LS, UK
} 
multiplication the Wiener functionals form an associative algebra and numerous subspaces of $\mathscr{W}$ are closed under this product, for example the Malliavin domain. A natural question arises: how is this algebraic structure manifested in $\mathscr{F}$ ? This is answered by the elegant formula

$$
f * g: \sigma \rightarrow \sum_{\alpha \subset \sigma} \int_{\Gamma\left(\mathbb{R}_{+}\right)} f(\alpha \cup \omega) g(\omega \cup \bar{\alpha}) d \lambda(\omega),
$$

where $\bar{\alpha}$ denotes the complement of $\alpha$ in $\sigma$. Dense subspaces of $\mathscr{F}$ which are stable under $*$ may be identified, and if $f$ and $g$ correspond to Wiener functionals $F$ and $G$ respectively, then $f * g$ corresponds to the pointwise product $F G$.

The form of the Wiener product (0.1) immediately suggests the following generalisation: replace the Lebesgue measure space $\mathbb{R}_{+}$by an abstract one $(S, m)$, $\left(\Gamma\left(\mathbb{R}_{+}\right), \lambda\right)$ by the symmetric measure space $(\Gamma(S), \mu)$ of $(S, m)$, and $*$ by the operation

$$
f *_{p} g: \sigma \rightarrow \sum_{\alpha \subset \sigma} \int_{\Gamma(S)} p(\omega, \alpha, \bar{\alpha}) f(\alpha \cup \omega) g(\omega \cup \bar{\alpha}) d \mu(\omega) .
$$

Incorporating an involution $\sim$ on $\Gamma(S)$, induced from a pointwise involution $i$ on $S$, we may further generalise:

$$
f \tilde{*}_{p} g: \sigma \rightarrow \sum_{\alpha \subset \sigma} \int_{\Gamma(S)} p(\omega, \alpha, \bar{\alpha}) f(\alpha \cup \tilde{\omega}) g(\omega \cup \bar{\alpha}) d \mu(\omega) .
$$

Such an approach unifies the various products considered by Maassen, Lindsay and Meyer. The Wiener product is implicit in [Maa] and is highlighted in [Me 1] where algebraic variations, including the Clifford product, are explored. The Bose product, extensively studied in [L M 1] is obtained by taking the measure space to be the sum of $\left(\mathbb{R}_{+}, m_{1}\right)$ and $\left(\mathbb{R}_{+}, m_{2}\right)$, where $m_{1}, m_{2}$ are non-zero multiples of Lebesgue measure, and the involution to be $\left(\sigma_{1}, \sigma_{2}\right)=\left(\sigma_{2}, \sigma_{1}\right)$. The Fermi product [L M 2] is obtained by a similar modification to the Bose product as is required to form the Clifford product from the Wiener product - namely the introduction of a $\{ \pm 1\}$-valued function $p$, which is dependent only on the relative position of the points of $\omega, \alpha$ and $\bar{\alpha}$ on the line, in (0.3) and (0.2) respectively.

The analysis of functions $p$ for which $(0.3)$ is associative rests on an analysis of the solutions of the functional equation

$$
q(\alpha, \beta) q(\alpha \cup \beta, \gamma)=q(\alpha, \beta \cup \gamma) q(\beta, \gamma) \quad \alpha, \beta, \gamma \in \Gamma(S) \text { disjoint }
$$

for non-vanishing complex-valued functions $q$. If $\Gamma$ and $\cup$ are replaced by a group $G$ and its binary operation, then (0.4) becomes the condition for $q$ to be a second order cocycle on $G$ with values in $\mathbb{C}^{\times}$, the group of non-zero complex numbers. Such objects arise in the theory of projective representations of groups, in particular they play a vital rôle in quantum theory [Var]. Our analysis has been inspired by the work of E. P. Wigner, V. Bargmann and G. W. Mackey [BaW, Bar, Mac].

The second order cocycles on $\Gamma(S)$ with values in $\mathbb{C}^{\times}$are classified in Sect. 2 and the associative products $*_{p}$ are described and classified in Sects. 3 and 4 . The pointwise product of Poisson functionals also gives rise to a product on Fock space [Me 1], [L M 2]. An analysis of deformations of this Poisson product, similar to $(0.2)$ and $(0.3)$ for the Wiener product, will appear elsewhere ([LP]).

Several authors, particularly P-A. Meyer, have raised the subject of noncommutative Poisson and Wiener type products [Me 2]. 
The last two sections give some applications of these ideas. In Sect. 6 operator fields, which are deformations of the quasi-free boson and fermion fields, are constructed in a natural way from cocycles and multipliers on $\Gamma$. These fields satisfy local commutation relations and provide bridges between boson and fermion fields - two such bridges are discussed. A continuum of inequivalent Levy fields [A P] with common covariance is also obtained, showing that commutation relations do not follow from martingale (fair game) assumptions alone $[P]$. In Sect. 7 we unify existence and uniqueness results for quantum stochastic differential equations by establishing the explicit form (cf. [Maa]) of the unique solution of linear stochastic differential equations driven by mixed noise.

\section{Set Notations}

For a set $S, \Gamma(S)$ or $\Gamma_{S}$ will denote the finite power set of $S:\{\alpha \subset S: \# \alpha<\infty\}$ which has the partition $\bigcup_{n=0}^{\infty} \Gamma_{n}(S)$, where $\Gamma_{n}(S)=\{\sigma \subset S: \# \sigma=n\}$. When $S$ is fixed and there is no danger of confusion we shall frequently drop mention of it in the notation, writing $\Gamma, \Gamma_{n}$ etc. The cartesian product $S^{n}$ will be understood to be the single point set with element 0 when $n=0$ and the collection of coordinates $\left\{s_{1}, \ldots, s_{n}\right\}$ of a typical element $\mathbf{s}=\left(s_{1}, \ldots, s_{n}\right) \in S^{n}$ will, by convention, by empty when $n=0$. To each element $\sigma$ of $\Gamma_{n}$ is associated $n$ ! points of $S^{n}$ - those points which have $\sigma$ as the set of their coordinates. $S^{(n)}(n=0,1, \ldots)$ will denote the subset of $S^{n}$ consisting of those points $\mathbf{s}$ with distinct coordinates: $s_{i} \neq s_{j}$ for $i \neq j$. To any function $f$ on $\Gamma(S)$ is naturally associated a sequence of symmetric functions $f_{n}$ on $S^{(n)}: f_{n}(\mathbf{s})=f\left(\left\{s_{1}, \ldots, s_{n}\right\}\right)$, and conversely such a sequence determines a function on $\Gamma(S)$. In particular, by considering indicator functions, there is a natural correspondence between subsets $E$ of $\Gamma(S)$ and sequences of symmetric subsets $E_{n}$ of $S^{(n)}$.

For each $n, \Gamma^{(n)}(S)$ will denote the subset

$$
\left\{\sigma=\left(\sigma_{1}, \ldots, \sigma_{n}\right) \in \Gamma(S)^{n}: \sigma_{i} \cap \sigma_{j}=\phi \text { if } i \neq j\right\}
$$

of $\Gamma(S)^{n}$, and $\Delta^{n}(S)$ will denote the set diagonal:

$$
\Gamma(S)^{n} \backslash \Gamma^{(n)}(S) \text {. }
$$

For $\alpha \in \Gamma^{(n)}, \alpha_{1} \cup \cdots \cup \alpha_{n}$ will be abbreviated to $|\alpha|$. In any summation over subsets $\alpha$ of an element $\sigma$ of $\Gamma, \bar{\alpha}$ will always denote the complement of $\alpha$ in $\sigma$.

$\mathscr{F}(A ; B)$ will denote the set of $B$-valued functions on $A$, and will be abbreviated to $\mathscr{F}(A)$ when $B=\mathbb{C}$. For a group valued function $f$ we call $\{a: f(a) \neq$ identity $\}$ the support of $f$. $\delta_{\varnothing}$ will always denote the function equal to one at $\varnothing$ and zero elsewhere in $\Gamma$, and an empty product will always be understood to be equal to one.

\section{Second Order Cohomology on $\Gamma(S)$}

In this section we describe the second order cohomology of $\Gamma(S)$ for an arbitrary set $S$. Since $S$ will be fixed we shall frequently drop references to it in the notation. A second order cocycle on $\Gamma(S)$, with values in the multiplicative group of non-zero 
complex numbers $\mathbb{C}^{\times}$, is a map $q: \Gamma^{(2)}(S) \rightarrow \mathbb{C}^{\times}$satisfying the cocycle identity

$$
q(\alpha, \beta) q(\alpha \cup \beta, \gamma)=q(\alpha, \beta \cup \gamma) q(\beta, \gamma), \quad(\alpha, \beta, \gamma) \in \Gamma^{(3)}(S) .
$$

The collection of such 2-cocycles forms an abelian group under the pointwise product which is also closed under complex conjugation and the involution

$$
\tilde{q}(\alpha, \beta)=q(\beta, \alpha) \text {. }
$$

If $f$ is a map from $\Gamma$ into $\mathbb{C}^{\times}$then

$$
\tau_{f}:(\alpha, \beta) \mapsto \frac{f(\alpha \cup \beta)}{f(\alpha) f(\beta)}, \quad(\alpha, \beta) \in \Gamma^{(2)}
$$

is called a trivial cocycle. For $f, g \in \mathscr{F}\left(\Gamma, \mathbb{C}^{\times}\right)$to determine the same trivial cocycle, $h=f / g$ must be multiplicative (homomorphic): $h(\alpha \cup \beta)=h(\alpha) h(\beta)$ for $(\alpha, \beta) \in \Gamma^{(2)}$. The trivial cocycles form a subgroup of the group of 2-cocycles. We denote the quotient group by $\mathscr{H}^{2}(\Gamma)$ and say that 2-cocycles $q_{1}, q_{2}$ are equivalent written $q_{1} \approx q_{2}$, if their ratio is trivial.

If $k$ is a map from $S^{(2)}$ to $\mathbb{C}^{\times}$then

$$
\pi_{k}:(\alpha, \beta) \mapsto \prod_{\substack{a \in \alpha \\ b \in \beta}} k(a, b), \quad(\alpha, \beta) \in \Gamma^{(2)}
$$

is called a product cocycle. Thus a 2-cocycle is a product cocycle if it is multiplicative in each argument.

Proposition 2.1. Let $k$ be a map from $S^{(2)}$ into $\mathbb{C}^{\times}$. If $k$ is symmetric then $\pi_{k}$ is trivial, and conversely.

Proof. If $k$ is symmetric, then the function $f: \Gamma \rightarrow \mathbb{C}^{\times}$,

$$
\alpha \mapsto \prod_{\{s, t\} \subset \alpha} k(s, t)
$$

is well defined and

$$
\tau_{f}(\alpha, \beta)=\frac{f(\alpha \cup \beta)}{f(\alpha) f(\beta)}=\prod_{a \in \alpha, b \in \beta} k(s, t)=\pi_{k}(\alpha, \beta) .
$$

In other words $\pi_{k}$ is trivial. The converse is obvious.

Putting $\beta=\varnothing$ in $(2.1)$ we see that $q(\varnothing, \cdot)$ and $q(\cdot, \varnothing)$ are equal constant functions on $\Gamma$; dividing by $q(\varnothing, \varnothing)$ therefore gives an equivalent 2-cocycle which is normalised: $q(\varnothing, \varnothing)=1$. (The equivalence is effected by the function equal to $q(\varnothing, \varnothing)$ at $\varnothing$ and 1 elsewhere). Although $\Gamma(S)$ is a semigroup under the union operation, and even a group under symmetric difference, all the 2-cocycles on these structures turn out to be trivial. Our less restrictive definition, only involving disjoint elements, is therefore needed for obtaining non-trivial cocycles.

When $\sigma=\left\{s_{1}, \ldots, s_{n}\right\}, \tau=\left\{t_{1}, \ldots, t_{m}\right\}$, where $(\sigma, \tau) \in \Gamma^{(2)}, q(\sigma, \tau)$ will sometimes be written $q\left(s_{1}, \ldots, s_{n} ; t_{1}, \ldots, t_{m}\right)$. The next result shows in particular that (normalized) 2-cocycles on $\Gamma$ are determined by their restrictions to $\Gamma_{1} \times \Gamma$, i.e. $\left\{(\{s\}, \tau) \in \Gamma^{(2)}\right\}$.

Proposition 2.2. Let $q$ be a 2-cocycle on $\Gamma(S)$ 
(i) $\operatorname{For}(\sigma, \tau) \in \Gamma^{(2)}$, if $\sigma=\left\{s_{1}, \ldots, s_{n}\right\} n \geqq 1$, then

$$
q(\sigma, \tau)=q\left(s_{n} ; \tau\right) \prod_{i=1}^{n-1} \frac{q\left(s_{i} ; \tau \cup\left\{s_{i+1}, \ldots, s_{n}\right\}\right)}{q\left(s_{i} ; s_{i+1}, \ldots, s_{n}\right)} .
$$

(ii) $q / \tilde{q}$ is a product cocycle, where $\tilde{q}$ is defined by (2.2).

\section{Proof.}

(i) Applying the cocycle identity to the triple $\left(\left\{s_{1}, \ldots, s_{n-1}\right\},\left\{s_{n}\right\}, \tau\right)$ we obtain

$$
q(\sigma, \tau)=q\left(s_{n} ; \tau\right) \frac{q\left(s_{1}, \ldots, s_{n-1} ; \tau \cup\left\{s_{n}\right\}\right)}{q\left(s_{1}, \ldots, s_{n-1} ; s_{n}\right)} .
$$

Iterating this identity for the numerator and denominator there is a cancellation of terms of the form $q\left(s_{1}, \ldots, s_{j} ; s_{j+1}\right)$ for $j=1, \ldots, n-2$, leaving the expression (2.5).

(ii) Let $(\alpha, \beta, \gamma) \in \Gamma^{(3)}$, then

so that

$$
\begin{aligned}
q(\alpha \cup \beta, \gamma) & =\frac{q(\beta, \gamma)}{q(\alpha, \beta)} q(\alpha, \gamma \cup \beta)=\frac{q(\beta, \gamma) q(\alpha, \gamma)}{q(\alpha, \beta) q(\gamma, \beta)} q(\gamma \cup \alpha, \beta) \\
& =\frac{q(\beta, \gamma) q(\alpha, \gamma) q(\alpha, \beta)}{q(\alpha, \beta) q(\gamma, \beta) q(\gamma, \alpha)} q(\gamma, \alpha \cup \beta),
\end{aligned}
$$

$$
q / \tilde{q}(\alpha \cup \beta, \gamma)=q / \tilde{q}(\alpha, \gamma) q / \tilde{q}(\beta, \gamma)
$$

By symmetry $q / \tilde{q}$ is also multiplicative in the second argument.

A 2-cocycle $q$ is symmetric if $\tilde{q}=q$ and skew-symmetric if $\tilde{q}=q^{-1}$. Clearly all trivial cocycles are symmetric, and a 2-cocycle is both symmetric and skewsymmetric if and only if it is (skew-)symmetric and $\{ \pm 1\}$-valued.

Proposition 2.3. Every 2-cocycle on $\Gamma(S)$ is equivalent to a skew-symmetric product cocycle.

Proof. Let $q$ be a 2-cocycle on $\Gamma$ which we suppose without loss to be normalised, and let $\pi$ be the product cocycle determined by the function $(s, t) \rightarrow q(s ; t)$. Define a sequence of function $f_{n}$ on $S^{(n)}, n=0,1,2, \ldots$ recursively by letting $f_{0}(0)=1$ and, for $n \geqq 1$,

$$
f_{n}:\left(s_{1}, \ldots, s_{n}\right) \mapsto f_{n-1}\left(s_{1}, \ldots, s_{n-1}\right)(q / \pi)\left(s_{n} ; s_{1}, \ldots, s_{n-1}\right) .
$$

$f_{1}(s)=f_{0}(0)(q / \pi)(s ; \phi)=1$ and, for $n \geqq 2$, the cocycle identity applied to the triple $\left(\left\{s_{n}\right\},\left\{s_{n-1}\right\},\left\{s_{1}, \ldots, s_{n-2}\right\}\right)$ yields

$$
\begin{aligned}
f_{n}\left(s_{1}, \ldots, s_{n}\right) & =f_{n-2}\left(s_{1}, \ldots, s_{n-2}\right)(q / \pi)\left(s_{n} ; s_{1}, \ldots, s_{n-1}\right)(q / \pi)\left(s_{n-1} ; s_{1}, \ldots, s_{n-2}\right) \\
& =f_{n-2}\left(s_{1}, \ldots, s_{n-2}\right)(q / \pi)\left(s_{n} ; s_{n-1}\right)(q / \pi)\left(s_{n-1}, s_{n} ; s_{1}, \ldots, s_{n-2}\right) \\
& =f_{n-2}\left(s_{1}, \ldots, s_{n-2}\right)(q / \pi)\left(s_{n-1}, s_{n} ; s_{1}, \ldots, s_{n-2}\right) .
\end{aligned}
$$

Thus, if $f_{j}$ is symmetric for $j<n$, by (2.6) $f_{n}$ is symmetric in its first $n-1$ arguments, and by (2.7) it is also symmetric in its last two arguments; in other words $f_{n}$ is symmetric. Since $f_{0}$ and $f_{1}$ are symmetric, it follows by induction that each of the 
functions $f_{n}$ is symmetric. The corresponding function $f$ on $\Gamma$ satisfies

$$
(q / \pi)(s ; \tau)=\frac{f(\tau \cup\{s\})}{f(\tau) f(\{s\})},
$$

so that $q=\tau_{f} \pi$. Now let $l$ be a logarithm of the function $(s, t) \rightarrow q(s ; t)$ on $S^{(2)}$, then $\pi=\pi_{k} \pi_{k^{\prime}}$ where $k=\exp (l-\tilde{l}) / 2$ and $k^{\prime}=\exp (l+\widetilde{l}) / 2$. Since $k^{\prime}$ is symmetric $\pi_{k^{\prime}}$ is trivial, $\tau_{g}$ say; on the other hand $k$ is skew-symmetric and $q=\tau_{f g} \pi_{k}$, so that the proof is complete.

\section{Corollary 2.4.}

(i) The trivial cocycles are the symmetric cocycles.

(ii) Two skew-symmetric product cocycles $\pi_{k_{1}}$ and $\pi_{k_{2}}$ are equivalent if and only if $k_{1} / k_{2}$ is skew-symmetric and $\{ \pm 1\}$-valued.

Since $\mathbb{C}^{\times}$and $\mathbb{C}^{\times} /\{ \pm 1\}$ are isomorphic groups the above degeneracy is not revealed in the second cohomology group of $\Gamma_{S}$.

\section{Theorem 2.5.}

$$
\mathscr{H}^{2}\left(\Gamma_{S}\right) \cong \mathscr{A}\left(S^{(2)} ; \mathbb{C}^{\times}\right)
$$

the multiplicative group of skew-symmetric $\mathbb{C}^{\times}$-valued functions on $S^{(2)}$.

In terms of any given partition $S_{1} \cup \cdots \cup S_{n}$ of $S$ we have the following alternative representation for 2-cocycles on $\Gamma_{S}$.

Proposition 2.6. Let $S_{1} \cup \cdots \cup S_{n}$ be a partition of $S$. Any 2-cocycle on $\Gamma_{S}$ is equivalent to a 2-cocycle of the form $\prod_{i \geqq j} q_{i j}$, where each $q_{i j}$ is a product cocycle, $(s, t) \rightarrow q_{i j}(s ; t)$ has support in $S_{i} \times S_{j} \cup S_{j} \times S_{i}$ and the $q_{i i}$ are skew-symmetric.

Proof. By Proposition 2.3 any 2-cocycle is equivalent to a skew-symmetric product cocycle $\pi$ which may be written $\prod_{i, j} \pi_{i j}$, where $\pi_{i j}(\alpha, \beta)=\pi\left(\alpha \cap S_{i}, \beta \cap S_{j}\right)$. Define a function $f$ and product cocycles $q_{i j}(i \geqq j)$ as follows:

$$
f(\alpha)=\prod_{i<j} \pi_{i j}(\alpha, \alpha) ; q_{i j}=\left\{\begin{array}{lll}
\pi_{i j} / \tilde{\pi}_{j i} & \text { if } & i>j \\
\pi_{i i} & \text { if } & i=j
\end{array} .\right.
$$

Then, by the multiplicativity of $\pi_{i j}$ (in each argument),

$$
\begin{aligned}
\left(\tau_{f} \prod_{i \geqq j} q_{i j}\right)(\alpha, \beta) & =\prod_{i<j} \frac{\pi_{i j}(\alpha \cup \beta, \alpha \cup \beta)}{\pi_{i j}(\alpha, \alpha) \pi_{i j}(\beta, \beta)} \frac{\prod_{i>j} \pi_{i j}(\alpha, \beta)}{\prod_{i<j} \pi_{i j}(\beta, \alpha)} \prod_{i} \pi_{i i}(\alpha, \beta) \\
& =\prod_{i, j} \pi_{i j}(\alpha, \beta),
\end{aligned}
$$

and the result follows.

Each 2-cocycle $q$ determines an algebraic structure on $\mathscr{F}\left(\Gamma_{s}\right)$ as follows:

$$
f \circ{ }_{q} g: \sigma \rightarrow \sum_{\alpha \in \sigma} q(\alpha, \bar{\alpha}) f(\alpha) g(\bar{\alpha})
$$


where $\bar{\alpha}$ denotes the complement of $\alpha$ in $\sigma: \sigma \backslash \alpha$. The cocycle identity expresses the associativity of this product. The resulting complex, associative algebra $\mathscr{F}\left(\Gamma_{S}, q\right)$ has unit $q(\varnothing, \varnothing)^{-1} \delta_{\varnothing}$ and is non-commutative unless $q$ is trivial.

$M_{f}$ will always denoted the pointwise multiplication operator $g \mapsto f g$ on complex valued functions defined on the same space as $f$.

Proposition 2.7. Let $q_{1}$ and $q_{2}$ be 2-cocycles on $\Gamma_{S}$ and $f \in \mathscr{F}\left(\Gamma_{S}\right)$. Then the following are equivalent:

(i) $q_{1} / q_{2}=\tau_{f}$,

(ii) $M_{f}: \mathscr{F}\left(\Gamma, q_{1}\right) \rightarrow \mathscr{F}\left(\Gamma, q_{2}\right)$ is an isomorphism.

Proof. Immediate.

Remark. Algebras of functions of several variables under products of the form

$$
f \circ{ }_{q} g(\boldsymbol{\sigma})=\sum q(\boldsymbol{\alpha}, \boldsymbol{\beta}) f(\boldsymbol{\alpha}) g(\boldsymbol{\beta}), \quad \boldsymbol{\sigma} \in \Gamma\left(S_{1}\right) \times \cdots \times \Gamma\left(S_{n}\right)
$$

(where the sum is over pairs $(\boldsymbol{\alpha}, \boldsymbol{\beta})$ for which each $\alpha_{i} \cup \beta_{i}$ is a partition of $\sigma_{i}$ ) may be reduced to the one variable case through the obvious identification of $\Gamma\left(S_{1}\right) \times \cdots \times \Gamma\left(S_{n}\right)$ with $\Gamma\left(S_{1} \Perp \cdots \Perp S_{n}\right)$.

When $q=(-1)^{n}$ where $n$ is defined, in terms of an ordering of $S$, by $n(\alpha, \beta)=\#\{(a, b) \in \alpha \times \beta: a>b\}, \mathscr{F}(\Gamma, q)$ is isomorphic to the algebra of antisymmetric functions on $\bigcup_{n=0}^{\infty} S^{(n)}$ under the antisymmetric product. When $q$ is the unit cocycle $\mathscr{F}$ is (naturally) isomorphic to the symmetric function algebra. Taking a cue from P-A. Meyer [Me 2] we call $\mathscr{F}\left(\Gamma_{S}, q\right)$ Wick-Grassmann algebras on $S$. By heeding the previous remark one can see that algebras of functions of several variables which are symmetric in some of the variables and anti-symmetric in the remaining variables are also covered by $(2.8)$.

\section{Wiener-Clifford Algebras}

In this section we construct associative algebras of measurable functions on $\Gamma(S, m)$, the symmetric measure space of a measure space $(S, m)$ [Gui], by means of three argument multipliers. Study of the isomorphism classes of these algebras shows that the generic product is obtained by a welding of two paradigm products: the Wiener product [Maa] and the Clifford product [Me 1].

Let $X=(S, m)$ be a $\sigma$-finite, non-atomic measure space. The correspondence between $\Gamma_{S}$ and $\bigcup_{n=0}^{\infty} S^{(n)}$ induces a measurable structure on $\Gamma_{S}: E \subset \Gamma_{S}$ is measurable if each $E_{n}$ is of the form $E_{n}^{\prime} \cap S^{(n)}$ for some $E_{n}^{\prime}$ which is measurable in the product space $X^{n}$. A measure $\mu_{X}$ is then defined by

$$
\mu_{X}(E)=\sum_{n=0}^{\infty}(n !)^{-1} m^{n}\left(E_{n}^{\prime}\right)
$$

where $m^{0}$ is point measure on $S^{0}$ and $m^{n}$ (for $n \geqq 1$ ) is the product measure on $S^{n}$. We denote the resulting measure space, the symmetric measure space of $X$, by $\Gamma(X)$ or $\Gamma_{X}$ and usually abbreviate $d \mu_{X}(\sigma)$ to $d \sigma$. 
Remark. Non-atomic $\sigma$-finite measures take all intermediate values between 0 and the (possibly infinite) total measure. It follows that each set diagonal $\Delta^{n}(S)$ is (contained in) a null set of the corresponding product measure. In particular $\mu_{X}$ is unambiguously defined.

The algebras considered here result from the convolution-like products:

$$
f *_{p} g: \sigma \rightarrow \sum_{\alpha \subset \sigma} \int p(\omega, \alpha, \bar{\alpha}) f(\alpha \cup \omega) g(\omega \cup \bar{\alpha}) d \omega,
$$

where the requirement of associativity imposes some restriction on the form of $p$.

From now on $\mathscr{F}(Y ; Z)$ will denote the space of measurable maps between the measure spaces $Y$ and $Z$. We introduce two further function spaces:

$$
\begin{aligned}
& \mathscr{F}_{r}\left(\Gamma^{n}\right)=\left\{f \in \mathscr{F}\left(\Gamma^{n} ; \mathbb{C}^{\times}\right): f \text { has relatively compact range and } f=1\right. \\
&\text { on the set diagonal } \left.\Delta^{n}(S)\right\}, \\
& \mathscr{K}(X)=\left\{f \in \mathscr{F}\left(\Gamma_{X}\right): \int a^{\# \sigma}|f(\sigma)|^{2} d \sigma<\infty \forall a>0\right\}=\bigcap_{a>0} \mathscr{D}\left(a^{N}\right),
\end{aligned}
$$

where $a^{N}$ is the multiplication operator: $\left(a^{N} f\right)(\sigma)=a^{\# \sigma} f(\sigma)$, on $L^{2}\left(\Gamma_{X}\right)$. The algebraic structures will be on $\mathscr{K}(X)$ and the products will be determined by functions from $\mathscr{F}_{r}\left(\Gamma^{3}\right)$. Recalling out set notations (Sect. 1) the following elementary identities are crucial:

\section{Proposition 3.1.}

(i) For $f \in \mathscr{F}\left(\Gamma^{(2)}\right),(\sigma, \tau) \in \Gamma^{(2)}$,

$$
\sum_{\gamma \subset \sigma \cup \tau} f(\gamma, \bar{\gamma})=\sum_{\alpha \subset \sigma} \sum_{\beta \subset \tau} f(\alpha \cup \beta, \bar{\alpha} \cup \bar{\beta}) .
$$

(ii) If $g \in \mathscr{F}\left(\Gamma^{n}\right)$ is integrable in the product measure $\mu^{n}$, then

$$
\int \cdots \int g\left(\sigma_{1}, \ldots, \sigma_{n}\right) d \sigma_{1} \cdots d \sigma_{n}=\int \sum_{|\alpha|=\sigma} g(\alpha) d \sigma .
$$

Proof. First suppose that $g$ is non-negative, and $\mathbf{j}=\left(j_{1}, \ldots, j_{n}\right) \in \mathbb{N}^{n}$ let $g_{\mathbf{j}}$ be the function on $S^{j_{1}} \times \cdots \times S^{j_{n}}=S^{j_{1}+\cdots+j_{n}}$ corresponding to $\left.g\right|_{\Gamma_{j 1} \times \cdots \times \Gamma_{j n}}$ which is symmetric in its first $j_{1}$ arguments, and in its second $j_{2}$ arguments, and so on. Then for $\left(s_{1}, \ldots, s_{N}\right) \in S^{(N)}$,

$$
\sum_{|\alpha|=\left\{s_{1}, \ldots, s_{N}\right\}} g\left(\alpha_{1}, \ldots, \alpha_{n}\right)=\sum_{j_{1}+\cdots+j_{n}=N}\left(j_{1} ! \cdots j_{n} !\right)^{-1} \sum_{\pi \in \mathscr{S}_{N}} g_{\mathrm{j}}\left(s_{\pi(1)}, \ldots, s_{\pi(N)}\right),
$$

so that, by the invariance of the product measure $m^{N}$ under (the natural action of) the permutation group $\mathscr{S}_{N}$, and regrouping courtesy of Fubini,

$$
\begin{aligned}
\int_{\Gamma_{N}} \sum_{|\alpha|=\sigma} g(\alpha) d \sigma & =(N !)^{-1} \sum_{j_{1}+\cdots+j_{n}=N}\left(j_{1} ! \cdots j_{n} !\right)^{-1} \sum_{\pi \in \mathscr{S}_{N}} \int \cdots \int g_{\mathbf{j}}\left(\mathbf{u}_{1}, \ldots, \mathbf{u}_{n}\right) d \mathbf{u}_{1} \cdots d \mathbf{u}_{n} \\
& =\sum_{j_{1}+\cdots+j_{n}=N} \int_{\Gamma_{j_{1}}} \cdots \int_{\Gamma_{j_{n}}} g\left(\sigma_{1}, \ldots, \sigma_{n}\right) d \sigma_{1} \cdots d \sigma_{n},
\end{aligned}
$$

where we have abbreviated $d m^{k}(\mathbf{u})$ to $d \mathbf{u}$. By summing over $N$ we obtain the identity (3.4). Since an integrable function is expressible as a linear combination of non-negative integrable functions (ii) follow by linearity. (i) is immediate.

Remark. Proposition 3.1 (ii) also follows by repeated application of the case $n=2$ 
which is proved in [L M 1]:

$$
\begin{aligned}
\int \sum_{|\alpha|=\sigma} f(\alpha) d \sigma & =\int \sum_{\beta \subset \sigma} \sum_{|\alpha|=\beta} f\left(\alpha_{1}, \ldots, \alpha_{n-1}, \bar{\beta}\right) d \sigma \\
& =\iint \sum_{|\alpha|=\beta} f\left(\alpha_{1}, \ldots, \alpha_{n-1}, \gamma_{n}\right) d \beta d \gamma_{n} \\
& \vdots \\
& =\int \cdots \int f\left(\gamma_{1}, \ldots, \gamma_{n}\right) d \gamma_{1}, \ldots, d \gamma_{n} .
\end{aligned}
$$

We have included a full proof here since much of the subsequent analysis depends on (3.4) which we shall refer to as the integral-sum identity/lemma.

Proposition 3.2. For $f, g \in \mathscr{K}(X)$ and $p \in \mathscr{F}\left(\Gamma^{3}\right)$ essentially bounded, $f *_{p} g$ is welldefined and satisfies the $L^{2}$-norm inequality

$$
\left\|a^{N}\left(f *_{p} g\right)\right\| \leqq \sup |p|\left\|(a \sqrt{3})^{N} f\right\|\left\|(a \sqrt{3})^{N} g\right\|, \quad a \geqq 1,
$$

while if $q \in \mathscr{F}\left(\Gamma^{2}\right)$ is essentially bounded and $f \circ{ }_{q} g$ is defined by (2.8),

$$
\left\|a^{N}\left(f \circ{ }_{q} g\right)\right\| \leqq \sup |q|\left\|(a \sqrt{2})^{N} f\right\|\left\|(a \sqrt{2})^{N} g\right\|, \quad a>0 .
$$

In particular, $f *_{p} g, f \circ_{q} g \in \mathscr{K}(X)$.

Proof. (cf. [L M 2]) An application of the integral sum identity (3.4) gives for $f \in \mathscr{K}(X)$,

$$
\int 3^{\# \sigma}|f(\sigma)|^{2} d \sigma=\int \sum_{\alpha \in \sigma} 2^{\# \alpha}|f(\sigma)|^{2} d \sigma=\iint 2^{\# \alpha}|f(\alpha \cup \beta)|^{2} d \alpha d \beta ;
$$

in other words, $f^{\alpha}: \beta \mapsto f(\alpha \cup \beta)$ is square integrable for almost all $\alpha$ and

$$
\left\|\sqrt{3}^{N} f\right\|^{2}=\int 2^{\# \alpha}\left\|f^{\alpha}\right\|^{2} d \alpha .
$$

By Cauchy-Schwartz and more applications of (3.4),

$$
\begin{aligned}
(\sup |p|)^{-2} \int\left|\left(a^{N}(f * g)\right)(\sigma)\right|^{2} d \sigma & \leqq \int\left\{\sum_{\alpha \subset \sigma} \int\left|\left(a^{N} f\right)(\alpha \cup \omega)\left(a^{N} g\right)(\omega \cup \bar{\alpha})\right| d \omega\right\}^{2} d \sigma \\
& \leqq \int 2^{\# \sigma} \sum_{\alpha \subset \sigma}\left\|\left(a^{N} f\right)^{\alpha}\right\|^{2}\left\|\left(a^{N} g\right)^{\bar{\alpha}}\right\|^{2} d \sigma \\
& =\int \sum_{\alpha \subset \sigma} 2^{\# \alpha}\left\|\left(a^{N} f\right)^{\alpha}\right\|^{2} 2^{\# \bar{\alpha}}\left\|\left(a^{N} g\right)^{\bar{\alpha}}\right\|^{2} d \sigma \\
& =\int 2^{\# \alpha}\left\|\left(a^{N} f\right)^{\alpha}\right\|^{2} d \alpha \int 2^{\# \beta}\left\|\left(a^{N} g\right)^{\beta}\right\|^{2} d \beta \\
& =\left\|(a \sqrt{3})^{N} f\right\|^{2}\left\|(a \sqrt{3})^{N} g\right\|^{2}
\end{aligned}
$$

in particular $f *_{p} g$ is defined almost everywhere and (3.5) holds. Again by the integral-sum lemma,

$$
\begin{aligned}
\left\|a^{N}\left(f \circ{ }_{q} g\right)\right\|^{2} & =\int\left|a^{\# \sigma} \sum_{\alpha \subset \sigma} q(\alpha, \bar{\alpha}) f(\alpha) g(\bar{\alpha})\right|^{2} d \sigma \\
& \leqq \int\left(2 a^{2}\right)^{\# \sigma} \sum_{\alpha \subset \sigma}|q(\alpha, \bar{\alpha}) f(\alpha) g(\bar{\alpha})|^{2} d \sigma
\end{aligned}
$$




$$
\begin{aligned}
& \leqq(\sup |q|)^{2} \iint\left(2 a^{2}\right)^{\# \alpha+\# \beta}|f(\alpha) g(\beta)|^{2} d \alpha d \beta \\
& =\left\{\sup |q|\left\|(a \sqrt{2})^{N} f\right\|\left\|(a \sqrt{2})^{N} g\right\|\right\}^{2},
\end{aligned}
$$

giving (3.6).

Proposition 3.3. Let $p \in \mathscr{F}_{r}\left(\Gamma^{3}\right)$ be essentially bounded and satisfy the identity

$$
\begin{aligned}
& p\left(\omega_{2} \cup \omega_{3}, \alpha_{1}, \alpha_{2} \cup \alpha_{3}\right) p\left(\omega_{1}, \alpha_{2} \cup \omega_{3}, \alpha_{3} \cup \omega_{2}\right) \\
& \quad=p\left(\omega_{1} \cup \omega_{2}, \alpha_{1} \cup \alpha_{2}, \alpha_{3}\right) p\left(\omega_{3}, \alpha_{1} \cup \omega_{2}, \alpha_{2} \cup \omega_{1}\right)
\end{aligned}
$$

for almost all $(\omega, \alpha) \in \Gamma^{6}$, then $*_{p}$ is associative.

Proof. By the non-atomicity of the measure $m,\{\sigma \in \Gamma: \sigma \cap \tau \neq \varnothing\}$ is a $\mu$-null set for each $\tau \in \Gamma$. Applying (3.3) with $(\sigma, \tau)=\left(\omega, \bar{\alpha}_{1}\right)$ and then (3.4) with $n=2$ and $\left(\alpha_{1}, \sigma\right)=\left(\omega_{2}, \omega\right)$ gives

$$
\begin{aligned}
f *(g * h)(\sigma)= & \sum_{\alpha \subset \sigma} \int p\left(\omega, \alpha_{1}, \bar{\alpha}_{1}\right) f\left(\omega \cup \alpha_{1}\right) g * h\left(\omega \cup \bar{\alpha}_{1}\right) d \omega \\
= & \sum_{\alpha_{1} \subset \sigma} \iint \sum_{\alpha_{2} \subset \bar{\alpha}_{1}} \sum_{\omega_{2} \subset \omega} p\left(\omega, \alpha_{1}, \bar{\alpha}_{1}\right) p\left(\omega_{1}, \bar{\omega}_{2} \cup \alpha_{2}, \omega_{2} \cup \bar{\alpha}_{2}\right) f\left(\alpha_{1} \cup \omega\right) \\
& \cdot g\left(\omega_{1} \cup \bar{\omega}_{2} \cup \alpha_{2}\right) h\left(\omega_{1} \cup \omega_{2} \cup \bar{\alpha}_{2}\right) d \omega d \omega_{1} \\
= & \sum_{|\alpha|=\sigma} \iiint p\left(\omega_{2} \cup \omega_{3}, \alpha_{1}, \alpha_{2} \cup \alpha_{3}\right) p\left(\omega_{1}, \omega_{3} \cup \alpha_{2}, \omega_{2} \cup \alpha_{3}\right) f\left(\alpha_{1} \cup \omega_{2} \cup \omega_{3}\right) \\
& \cdot g\left(\omega_{1} \cup \alpha_{2} \cup \omega_{3}\right) h\left(\omega_{1} \cup \omega_{2} \cup \alpha_{3}\right) d \omega_{1} d \omega_{2} d \omega_{3} .
\end{aligned}
$$

On the other hand, applying (3.3) with $(\sigma, \tau)=\left(\omega, \bar{\alpha}_{3}\right)$ and then (3.4) with $\left(\alpha_{1}, \sigma\right)=\left(\omega_{2}, \omega\right)$ gives for $(f * g) * h(\sigma)$,

$$
\begin{aligned}
& \sum_{\alpha_{3} \subset \sigma} \int p\left(\omega, \bar{\alpha}_{3}, \alpha\right) f * g\left(\omega \cup \bar{\alpha}_{3}\right) h\left(\omega \cup \alpha_{3}\right) d \omega \\
& =\sum_{\alpha_{\mathcal{s}}=\sigma} \iint \sum_{\bar{\alpha}_{2} \subset \alpha_{3}} \sum_{\omega_{2} \subset \omega} p\left(\omega, \bar{\alpha}_{3}, \alpha_{3}\right) p\left(\omega_{3}, \omega_{2} \cup \bar{\alpha}_{2}, \bar{\omega}_{2} \cup \bar{\alpha}_{2}\right) \\
& \quad \cdot f\left(\omega_{3} \cup \omega_{2} \cup \bar{\alpha}_{2}\right) g\left(\omega_{3} \cup \bar{\omega}_{2} \cup \alpha_{2}\right) h\left(\omega \cup \alpha_{3}\right) d \omega d \omega_{3} \\
& =\sum_{|\alpha|=\sigma} \iiint p\left(\omega_{1} \cup \omega_{2}, \alpha_{1} \cup \alpha_{2}, \alpha_{3}\right) p\left(\omega_{3}, \omega_{2} \cup \alpha_{1}, \omega_{1} \cup \alpha_{2}\right) \\
& \quad \cdot f\left(\alpha_{1} \cup \omega_{2} \cup \omega_{3}\right) g\left(\omega_{1} \cup \alpha_{2} \cup \omega_{3}\right) h\left(\omega_{1} \cup \omega_{2} \cup \alpha_{3}\right) d \omega_{1} d \omega_{2} d \omega_{3} .
\end{aligned}
$$

Thus if $p$ satisfies $(3.8) *_{p}$ is associative.

Thus the a.e. equality (3.8) is a sufficient condition on an essentially bounded $p$ for the associativity of $*_{p}$. When $X$ is a separable measure space the condition is also sufficient. Since the proof does not illuminate the rest of the paper, where separability plays no rôle, we defer it to [LP]. In view of $(3.5) *_{p}$ descends to the quotient of $\mathscr{K}(X)$ by the $\mu_{X}$-null functions. By abuse of notation we also denote the quotient by $\mathscr{K}(X)$.

Elements $p$ of $\mathscr{F}_{r}\left(\Gamma^{3}\right)$, satisfying (3.8) for all $(\omega, \alpha)$ in $\Gamma^{(6)}$ will be called multipliers. We do not know the answer to the following natural question: suppose that $p$ belongs to $\mathscr{F}_{r}\left(\Gamma^{3}\right)$ and $*_{p}$ is associative, then does $p$ agree almost everywhere with a multiplier on $X$ ? A positive answer to this would permit a more liberal definition of multipliers. 
The first interesting non-trivial multiplier is the Clifford multiplier given by

$$
p(\omega, \alpha, \beta)=(-1)^{n(\omega \cup \beta, \omega \cup \alpha)},
$$

where $n(\sigma, \tau)=\#\{(s, t) \sigma \times \tau: s>t\}$ and $>$ is some measurable ordering on $X$. We shall call pairs $\left(\mathscr{K}(X), *_{p}\right)$ Wiener-Clifford algebras on $X$.

Proposition 3.4. $p$ is a multiplier if and only if there are $f \in \mathscr{F}_{r}(\Gamma)$ and $q \in \mathscr{F}_{r}\left(\Gamma^{2}\right)$ such that

(i) $q$ satisfies the cocycle identity (2.1),

(ii) $\tau_{f} q \tilde{q}=\tau_{f}^{2} q^{4} \equiv 1$,

(iii) $p(\omega, \alpha, \beta)=f(\omega) \frac{q(\alpha, \beta)}{q(\alpha, \omega) q(\omega, \beta)} \forall(\omega, \alpha, \beta) \in \Gamma^{(3)}$.

Proof. Since $p$ satisfies (3.8) if and only if non-zero multiples of $p$ do, and since such multiples may be absorbed by $f$ in (3.11), we may assume $p$ to be normalised: $p(\phi, \phi, \phi)=1$. Suppose $p$ is a multiplier and define $q$ and $f$ by

$$
q(\alpha, \beta)=p(\phi, \alpha, \beta) ; \quad f(\omega)=p(\omega, \phi, \phi) .
$$

Putting $\omega=\varnothing$ in (3.8) we see that (up to a null set) $q$ is a 2-cocycle, and putting $\alpha=\varnothing$ we obtain the cyclic relation

$$
f\left(\omega_{2} \cup \omega_{3}\right) p\left(\omega_{1}, \omega_{3}, \omega_{2}\right)=f\left(\omega_{1} \cup \omega_{2}\right) p\left(\omega_{3}, \omega_{2}, \omega_{1}\right) .
$$

Putting $\alpha_{2}=\alpha_{3}=\omega_{2}=\omega_{3}=\varnothing$ in (3.8) and applying (3.13) gives

so that

$$
f(\omega)=p(\omega, \alpha, \varnothing) q(\alpha, \omega)=\frac{f(\omega \cup \alpha)}{f(\alpha)} q(\omega, \alpha) q(\alpha, \omega),
$$

$$
\tau_{f} q \tilde{q}=1
$$

Putting $\omega_{3}=\varnothing$ in (3.13) and using (3.14) gives

$$
p\left(\omega_{1}, \varnothing, \omega_{2}\right)=\frac{f\left(\omega_{1} \cup \omega_{2}\right)}{f\left(\omega_{2}\right)} q\left(\omega_{2}, \omega_{1}\right)=\frac{f\left(\omega_{1}\right)}{q\left(\omega_{1}, \omega_{2}\right)} .
$$

Putting $\alpha_{1}=\alpha_{2}=\omega_{2}=\varnothing$ in (3.8) and applying (3.15) now yields

$$
p\left(\omega_{1}, \omega_{3}, \alpha_{3}\right)=\frac{p\left(\omega_{1}, \varnothing, \alpha_{3}\right) p\left(\omega_{3}, \varnothing, \omega_{1}\right)}{p\left(\omega_{3}, \varnothing, \alpha_{3}\right)}=\frac{f\left(\omega_{1}\right) f\left(\omega_{3}\right)}{f\left(\omega_{3}\right)} \frac{q\left(\omega_{3}, \alpha_{3}\right)}{q\left(\omega_{1}, \alpha_{3}\right) q\left(\omega_{3}, \omega_{1}\right)} .
$$

Thus $p$ is composed from a function $f$ and a 2-cocycle $q$, which stand in the reciprocal relation (3.14) through the formula (3.11). It remains to show that a function $p$ given by such a pair $(q, f)$ through (3.11) is a multiplier if and only if $\tau_{f}^{2} q^{4} \equiv 1$. Let $p$ be such a function, then the ratio

$$
\frac{p\left(\omega_{2} \cup \omega_{3}, \alpha_{1}, \alpha_{2} \cup \alpha_{3}\right) p\left(\omega_{1}, \alpha_{2} \cup \omega_{3}, \alpha_{3} \cup \omega_{2}\right)}{p\left(\omega_{1} \cup \omega_{2}, \alpha_{1} \cup \alpha_{2}, \alpha_{3}\right) p\left(\omega_{3}, \alpha_{1} \cup \omega_{2}, \alpha_{2} \cup \omega_{1}\right)}
$$

may be expressed as a product $P_{1} P_{2} P_{3}$, where

$$
P_{1}=\frac{f\left(\omega_{2} \cup \omega_{3}\right) f\left(\omega_{1}\right)}{f\left(\omega_{1} \cup \omega_{2}\right) f\left(\omega_{3}\right)}
$$




$$
\begin{aligned}
P_{2} & =\frac{q\left(\alpha_{1}, \alpha_{2} \cup \alpha_{3}\right)}{q\left(\alpha_{1} \cup \alpha_{2}, \alpha_{3}\right)} \frac{q\left(\omega_{1} \cup \omega_{2}, \alpha_{3}\right)}{q\left(\omega_{1}, \alpha_{3} \cup \omega_{2}\right)} \frac{q\left(\alpha_{1} \cup \omega_{2}, \omega_{3}\right)}{q\left(\alpha_{1}, \omega_{2} \cup \omega_{3}\right)} \frac{q\left(\omega_{3}, \alpha_{2} \cup \omega_{1}\right)}{q\left(\alpha_{2} \cup \omega_{3}, \omega_{1}\right)} \\
& =\frac{q\left(\alpha_{1}, \alpha_{2}\right)}{q\left(\alpha_{2}, \alpha_{3}\right)} \frac{q\left(\omega_{2}, \alpha_{3}\right)}{q\left(\omega_{1}, \omega_{2}\right)} \frac{q\left(\omega_{2}, \omega_{3}\right)}{q\left(\alpha_{1}, \omega_{2}\right)} \frac{q\left(\omega_{3}, \alpha_{2}\right)}{q\left(\alpha_{2}, \omega_{1}\right)} \\
P_{3} & =\frac{q\left(\alpha_{2} \cup \omega_{3}, \alpha_{3} \cup \omega_{2}\right) q\left(\alpha_{1} \cup \alpha_{2}, \omega_{1} \cup \omega_{2}\right)}{q\left(\alpha_{1} \cup \omega_{2}, \alpha_{2} \cup \omega_{1}\right) q\left(\omega_{2} \cup \omega_{3}, \alpha_{2} \cup \alpha_{3}\right)} .
\end{aligned}
$$

Moreover, by (3.14)

$$
P_{1} P_{2}=\frac{q\left(\omega_{2}, \omega_{1}\right) q\left(\alpha_{1}, \alpha_{2}\right) q\left(\omega_{2}, \alpha_{3}\right) q\left(\omega_{3}, \alpha_{2}\right)}{q\left(\omega_{3}, \omega_{2}\right) q\left(\alpha_{2}, \alpha_{3}\right) q\left(\alpha_{1}, \omega_{2}\right) q\left(\alpha_{2}, \omega_{1}\right)} .
$$

Applying the cocycle identity successively to the triples $\left(\alpha_{1} \cup \omega_{2}, \alpha_{2}, \omega_{1}\right),\left(\alpha_{1} \cup \alpha_{2}\right.$, $\left.\omega_{2}, \omega_{1}\right)$ and $\left(\omega_{2}, \alpha_{1}, \alpha_{2}\right)$, and using the fact that $q / \tilde{q}$ is a product cocycle,

$$
\begin{aligned}
\frac{q\left(\alpha_{1} \cup \alpha_{2}, \omega_{1} \cup \omega_{2}\right)}{q\left(\alpha_{1} \cup \omega_{2}, \alpha_{2} \cup \omega_{1}\right)} & =\frac{q\left(\alpha_{1} \cup \alpha_{2}, \omega_{1} \cup \omega_{2}\right)}{q\left(\alpha_{1} \cup \alpha_{2} \cup \omega_{2}, \omega_{1}\right)} \frac{q\left(\alpha_{2}, \omega_{1}\right)}{q\left(\alpha_{1} \cup \omega_{2}, \alpha_{2}\right)} \\
& =\frac{q\left(\alpha_{1} \cup \alpha_{2}, \omega_{2}\right)}{q\left(\omega_{2}, \omega_{1}\right)} \frac{q\left(\alpha_{2}, \omega_{1}\right)}{q\left(\omega_{2}, \alpha_{1} \cup \alpha_{2}\right)} \frac{q\left(\omega_{2}, \alpha_{1}\right)}{q\left(\alpha_{2}, \alpha_{1}\right)} \\
& =\frac{q\left(\alpha_{2}, \omega_{1}\right) q\left(\omega_{2}, \alpha_{1}\right)}{q\left(\omega_{2}, \omega_{1}\right) q\left(\alpha_{1}, \alpha_{2}\right)} q / \tilde{q}\left(\alpha_{1}, \omega_{2}\right) q / \tilde{q}\left(\alpha_{2}, \omega_{2}\right) \\
& =\frac{q\left(\alpha_{2}, \omega_{1}\right) q\left(\alpha_{1}, \omega_{2}\right)}{q\left(\omega_{2}, \omega_{1}\right) q\left(\alpha_{1}, \alpha_{2}\right)} q / \tilde{q}\left(\alpha_{2}, \omega_{2}\right),
\end{aligned}
$$

and similarly,

$$
\frac{q\left(\alpha_{2} \cup \omega_{2}, \alpha_{3} \cup \omega_{2}\right)}{q\left(\omega_{2} \cup \omega_{3}, \alpha_{2} \cup \alpha_{3}\right)}=\frac{q\left(\alpha_{2}, \alpha_{3}\right) q\left(\omega_{3}, \omega_{2}\right)}{q\left(\omega_{2}, \alpha_{3}\right) q\left(\omega_{3}, \alpha_{2}\right)} q / \tilde{q}\left(\alpha_{2}, \omega_{3}\right) .
$$

Therefore the ratio $(3.16)$ is $(q / \tilde{q})^{2}\left(\alpha_{2}, \omega_{2}\right)$ or by (3.14),

$$
\tau_{f}^{2}\left(\alpha_{2}, \omega_{2}\right) q^{4}\left(\alpha_{2}, \omega_{2}\right)
$$

Thus the identity (3.8) holds for a function $p$ given by (3.11), where $q$ is a 2-cocycle and $\tau_{f} q \tilde{q} \equiv 1$, if and only if $\tau_{f}^{2} q^{4} \equiv 1$, and the proof is complete.

A function $f: \Gamma_{S} \rightarrow \mathbb{C}$ is called multiplicative if

$$
f(\alpha \cup \beta)=f(\alpha) f(\beta) \text { for } \alpha, \beta \text { disjoint. }
$$

Thus multiplicative functions are of the form

$$
\sigma \rightarrow \prod_{s \in \sigma} \phi(s)
$$

where $\phi$ is a function on $S$. Notation: $\varepsilon_{\phi}$. If $\phi_{1}$ and $\phi_{2}$ are measurable functions on $X$ which agree a.e. $m$, then $\varepsilon_{\phi_{1}}=\varepsilon_{\phi_{2}}$ a.e. $\mu$. If $\phi \in L^{1}(X)$ then $\varepsilon_{\phi} \in L^{1}\left(\Gamma_{X}\right)$ and $\int \varepsilon_{\phi} d \mu=\exp \left\{\int \phi d m\right\}$. When $\phi \in L^{2}(X) \varepsilon_{\phi}$ is called the exponential vector determined by $\phi$.

Note that a product cocycle in $\mathscr{F}_{r}\left(\Gamma^{2}\right)$ must be of unit modulus (outside a null set in $\Gamma^{(2)}$ ). 
Lemma 3.5. Let $q \in \mathscr{F F}_{r}\left(\Gamma^{2}\right)$ be a 2-cocycle and $f \in \mathscr{F}_{r}\left(\Gamma^{1}\right)$, then the following are equivalent:

(i) $\tau_{f} q \tilde{q}=\tau_{f}^{2} q^{4} \equiv 1$,

(ii) $q=\tau_{h} \pi_{k}$, where $k \tilde{k}=1, k\left(S^{2}\right) \subset\{1, i,-i\}$ and $f h^{2}$ is multiplicative.

Proof. Suppose that $q$ is of the form (ii), then $\tau_{f} q \tilde{q}=\tau_{f h^{2}} \pi_{k} \tilde{\pi}_{k}=\tau_{f h^{2}} \equiv 1$ and $\tau_{f}^{2} q^{4}=\left(\tau_{f h^{2}}\right)^{2}\left(\pi_{k}\right)^{4} \equiv 1$, so (ii) implies (i). Suppose now that $q$, $f$ satisfy (i), let $g_{0}$ be a measurable square root of $f$ and let $\eta=\tau_{g_{0}} q$. Then

$$
\eta \tilde{\eta}=\tau_{f} q \tilde{q} \equiv 1 ; \quad \eta^{4}=\tau_{f^{2}} q^{4} \equiv 1,
$$

so that $\eta$ is skew-symmetric with range in $\{1, i,-1,-1\}$. Let $k_{1}(s, t)=\eta(s, t)$, then $\eta=\tau_{g_{1}} \pi_{k_{1}}$ for some $g_{1} \in \mathscr{F}_{r}\left(\Gamma^{1}\right)$. Define $k_{2}$ by

$$
k_{2}(s, t)=\left\{\begin{aligned}
-1, & \text { if } k_{1}(s, t)=-1 \\
1, & \text { otherwise. }
\end{aligned}\right.
$$

Then, since $k_{2}$ is symmetric, $\pi_{k_{2}}$ is trivial: $\tau_{g_{2}}$, say. Letting $h=g_{0}^{-1} g_{1} g_{2}$ and $k=k_{1} k_{2}$ we have $q=\tau_{h} \pi_{k}$ and

$$
1 \equiv \tau_{f} q \tilde{q}=\tau_{f h^{2}} \pi_{k \tilde{k}}=\tau_{f h^{2}},
$$

so that $f h^{2}$ is multiplicative. Since $k$ has range in $\{1, i,-i\}$ the proof is complete.

If $g \in \mathscr{F}\left(X ; \mathbb{C}^{\times}\right)$and $\varepsilon$ is the multiplicative function determined by a measurable square root of $g$, then $\varepsilon_{g}(\omega)=\varepsilon(\alpha \cup \beta)^{-1} \varepsilon(\alpha \cup \omega) \varepsilon(\omega \cup \beta)$. By letting $g$ be the function $s \mapsto\left(f h^{2}\right)(\{s\})$, Lemma 3.5 combines with Proposition 3.4 to give:

Proposition 3.6. Let $p$ be a multiplier on $\Gamma_{X}$, then

$$
p(\omega, \alpha, \beta)=\frac{f(\alpha \cup \omega) f(\omega \cup \beta)}{f(\alpha \cup \beta)} \frac{\pi(\alpha, \beta)}{\pi(\alpha, \omega) \pi(\omega, \beta)},
$$

where $f \in \mathscr{F}_{r}\left(\Gamma^{1}\right)$ and $\pi=\pi_{k}$, where $k \in \mathscr{F}\left(S^{2}\right)$ is skew-symmetric with range in $\{1, \pm i\}$.

Note that the functions $k$ that arise in (3.17) are all the functions of the form

$$
k_{F}:(s, t) \rightarrow\left\{\begin{aligned}
i & \text { on } F \\
-i & \text { on } \tilde{F} \\
1 & \text { elsewhere }
\end{aligned}\right.
$$

where $F$ is a (measurable) subset of $S^{(2)}$ satisfying $F \cap \tilde{F}=\varnothing$.

Writing $p=(f, k)$ for a multiplier with representation (3.17) we define equivalence for multipliers by $(f, k) \approx\left(f^{\prime}, k^{\prime}\right)$ if $\pi_{k}$ and $\pi_{k}$, are equivalent cocycles, modulo a null set-in other words $\pi_{k^{\prime}}=\tau_{g} \pi_{k}$ a.e. for some $g \in \mathscr{F}_{r}\left(\Gamma^{1}\right)$. We denote the group of equivalence classes of multipliers by $\mathscr{M}\left(\Gamma_{X}\right)$. The justification for this definition of equivalence will come later.

Proposition 3.7. Let $p=(f, k), p^{\prime}=\left(f^{\prime}, k^{\prime}\right)$ be multipliers on $\Gamma_{X}$. Then the following are equivalent:

(i) $p \approx p^{\prime}$,

(ii) $\operatorname{supp} k \Delta \operatorname{supp} k^{\prime}$ is null. 
Proof. Let $E$ be the symmetric set supp $k \Delta \operatorname{supp} k^{\prime}$ and let $k_{1}$ be the symmetric function agreeing with $k^{\prime} k^{-1}$ outside $E$ and equal to 1 on $E$. Then $\pi_{k_{1}}$ agrees with $\pi_{k^{\prime}} \pi_{k}^{-1}$ outside the set $\hat{E}=\{(\{s\} \cup \alpha,\{t\} \cup \beta):(s, t) \in E, \alpha, \beta \in \Gamma\}$. Since $\hat{E}$ is $\mu^{2}$-null if and only if $E$ is $m^{2}$-null the result follows

Theorem 3.8.

$$
\mathscr{M}\left(\Gamma_{X}\right) \cong\left(\mu\left(\Gamma_{2}\right), \Delta\right)
$$

the measure algebra of $\Gamma_{2}$ considered as a group under symmetric difference.

Proof. By Proposition 3.7, $\Phi:[(f, k)] \rightarrow[\operatorname{supp} k]$ is a well-defined injective map from $\mathscr{M}\left(\Gamma_{X}\right)$ to the equivalence classes of symmetric measurable subsets of $S^{(2)}$. Let $E$ be such a subset of $S^{(2)}$ then, if $F \cup \widetilde{F}$ is a measurable partition of $E . \Phi\left(\left[\left(1, k_{F}\right)\right]\right)=[E]$, so $\Phi$ is bijective. Now suppose that $E=E_{1} \Delta E_{2}$, where $E_{1}, E_{2}$ are symmetric subsets of $S^{(2)}$, and let $F \cup \widetilde{F}$ be a measurable partition of $E$ and $F_{3} \cup \tilde{F}_{3}$ a measurable partition of $E_{1} \cap E_{2}$. Define $k_{1}=k_{F_{3} \cup F_{1}}$ and $k_{2}=k_{\tilde{F}_{2} \cup F_{1}}$, where $F_{i}=F \cap E_{i}(i=1,2)$, then $\operatorname{supp} k_{i}=E_{i}$ and $\operatorname{supp} k_{1} k_{2}=E$. Thus $\Phi^{-1}\left(\left[E_{1} \Delta E_{2}\right]\right)=\Phi^{-1}\left(\left[E_{1}\right]\right) \Phi^{-1}\left(\left[E_{2}\right]\right)$, and the theorem now follows since the measure algebra of $\Gamma_{2}$ is isomorphic to the symmetric measure algebra of $X^{(2)}$.

Suppose that $p=(f, k)$ and $p^{\prime}=\left(f^{\prime}, k^{\prime}\right)$ are equivalent multipliers so that $\pi_{k^{\prime}}=\tau_{h} \pi_{k}$ almost everywhere and, for a.a. $\sigma, h(\sigma)=\prod_{\{s, t\} \subset \sigma}\left(k / k^{\prime}\right)(s, t)$ and is $\{ \pm 1\}-$ valued. Then $M_{h f / f^{\prime}}$ is an isomorphism from $\left(\mathscr{K}(X), *_{p}\right)$ to $\left(\mathscr{K}(X), *_{p^{\prime}}\right)$, justifying our notion of equivalence for multipliers. Again Wiener-Clifford algebras of functions of several variables may be reduced to one variable function algebras via the natural identification of $\Gamma\left(X_{1}\right) \times \cdots \times \Gamma\left(X_{n}\right)$ with $\Gamma(X)$, where $X$ is the sum of the measure spaces $X_{1}, \ldots, X_{n}$. In particular super-symmetric WienerClifford algebras arise by choosing multipliers $p$ of the form $(\omega, \alpha, \beta) \rightarrow \prod_{i} p\left(\omega \cap S_{i}\right.$, $\left.\alpha \cap S_{i}, \beta \cap S_{i}\right)$, where $\left.P\right|_{\Gamma^{(3)}\left(S_{i}\right)}$ is a Clifford multiplier for $i=1, \ldots, k$, say, and the Wiener multiplier ( $\equiv 1$ ) for $i=k+1, \ldots, n$.

\section{Twisted Wiener-Clifford Algebras}

In this section we construct and classify a further class of algebras by means of an involution on $S$, that is a map $i: S \rightarrow S$ whose square is the identity map. The Bose and Fermi products, which are discussed extensively in [L M 1], are included and also some interesting new products. The results of this section subsume those of the previous one since the involution may be the identity map.

Let $Z=\left(S, m, i, S_{+}\right)$, where $X=(S, m)$ is a $\sigma$-finite, non-atomic measure space, $i$ is a measurable involution on $S$ and $S_{+}$is a measurable subset of $S$ such that if $S_{0}=\{s \in S: i(s)=s\}$ and $S_{-}=i\left(S_{+}\right)$, then $S_{0} \cup S_{+} \cup S_{-}$is a partition of $S$. Let $\sim$ be the involution on $\Gamma^{n}(S)$ induced by $i$ : $\tilde{\sigma}=\left(\tilde{\sigma}_{n}, \ldots, \tilde{\sigma}_{1}\right)$, where $\left\{s_{1}, \ldots, s_{n}\right\} \sim \mathcal{\sim}=\left\{i\left(s_{1}\right), \ldots\right.$, $\left.i\left(s_{n}\right)\right\}$, and for $f \in \mathscr{F}\left(\Gamma^{n}\right)$ let $\tilde{f}$ be the induced map: $\tilde{f}(\sigma)=f(\tilde{\boldsymbol{\sigma}})$. Note that this does not conflict with the previous use of $\sim$. For $Z$ as above, $\Gamma(Z)$ or $\Gamma_{Z}$ will denote the measure space $\Gamma(S, m)$ together with this extra structure. $\sim$ (and $i$ ) will be referred to as the twist. 
We are interested in those elements $p$ of $\mathscr{F}_{r}\left(\Gamma^{3}\right)$ for which the twisted convolution

$$
f \tilde{*}_{p} g: \sigma \mapsto \sum_{\alpha \subset \sigma} \int p(\omega, \alpha, \bar{\alpha}) f(\alpha \cup \tilde{\omega}) g(\omega \cup \bar{\alpha}) d \omega
$$

defines an associative product on $\mathscr{K}(Z):=\mathscr{K}(S, m+\tilde{m})$. A similar inequality to (3.5) holds so that $\mathscr{K}(Z)$ is closed under $\tilde{*}_{p}$ which again descends to the quotient modulo $\mu_{X}$-null functions.

Proposition 4.1. For $p \in \mathscr{F}_{r}\left(\Gamma^{3}\right)$, let $D_{p} \in \mathscr{F}\left(\Gamma^{6}\right)$ be given by

$$
\begin{aligned}
(\boldsymbol{\alpha}, \boldsymbol{\omega}) \mapsto & p\left(\omega_{2} \cup \omega_{3}, \alpha_{1}, \alpha_{2} \cup \alpha_{3}\right) p\left(\omega_{1}, \omega_{3} \cup \alpha_{2}, \omega_{2} \cup \alpha_{3}\right) \\
& -p\left(\omega_{1} \cup \omega_{2}, \alpha_{1} \cup \alpha_{2}, \alpha_{3}\right) p\left(\omega_{3}, \alpha_{1} \cup \tilde{\omega}_{2}, \alpha_{2} \cup \tilde{\omega}_{1}\right),
\end{aligned}
$$

then the vanishing of $D_{p}$ (a.e.) is sufficient for the associativity of $\tilde{*}_{p}$ on $\mathscr{K}(Z)$.

Proof. The same applications of Lemma 3.1 as were used in the proof of Proposition 3.2 lead to the expression

$$
\sum_{|\alpha|=\sigma} \iiint D_{p}(\boldsymbol{\alpha}, \boldsymbol{\omega}) f\left(\alpha_{1} \cup \tilde{\omega}_{2} \cup \tilde{\omega}_{3}\right) g\left(\tilde{\omega}_{1} \cup \alpha_{2} \cup \omega_{3}\right) h\left(\omega_{1} \cup \omega_{2} \cup \alpha_{3}\right) d \omega_{1} d \omega_{2} d \omega_{3}
$$

for $\{f \tilde{*}(g \tilde{*} h)-(f \tilde{*} g) \tilde{*} h\}(\sigma)$ and the result follows.

Elements $p$ of $\mathscr{F}_{r}\left(\Gamma_{Z}^{3}\right)$ for which $D_{p}$ vanishes identically will be called multipliers on $\Gamma_{Z}$. In our analysis of multipliers on $\Gamma_{Z}$ we continue to denote by $\tau_{f}$ and $\pi_{k}$ the trivial and product cocycles determined by $f \in \mathscr{F}_{r}\left(\Gamma^{1}\right)$ and $k \in \mathscr{F}_{r}\left(S^{2}\right)$ respectively.

Proposition 4.2. $p$ is a multiplier on $\Gamma_{Z}$ if and only if there are $f \in \mathscr{F}_{r}\left(\Gamma^{1}\right)$ and $q \in \mathscr{F}_{r}\left(\Gamma^{2}\right)$ such that

(i) q satisfies the cocycle identity (2.1),

(ii) $\tau_{f} q \tilde{q}=1$,

(iii) $\frac{q(\alpha, \beta) q(\alpha, \tilde{\beta})}{q(\beta, \alpha) q(\widetilde{\beta}, \alpha)}=1$,

(iv) $p(\omega, \alpha, \beta)=f(\omega) \frac{q(\alpha, \beta)}{q(\alpha, \tilde{\omega}) q(\omega, \beta)} \quad \forall(\omega, \alpha, \beta) \in \Gamma^{(3)}$.

Proof. Applying identical steps to (4.2) as were applied to (3.7) in the proof of Proposition 3.4 leads to the identities (4.3) and (4.5), $q$ being a 2-cocycle on $\Gamma(S)$. Further identical steps lead to the expression

$$
\frac{q\left(\alpha_{2}, \tilde{\omega}_{2}\right) q\left(\alpha_{2}, \omega_{2}\right)}{q\left(\tilde{\omega}_{2}, \alpha_{2}\right) q\left(\omega_{2}, \alpha_{2}\right)}
$$

for the ratio (3.16) ${ }^{\sim}$. Thus $p$ is a multiplier on $\Gamma_{Z}$ if and only if $p$ is of the form (4.5), where $(q, f)$ satisfies (4.3) and $q$ is a 2-cocycle satisfying (4.4).

Lemma 4.3. Let $q \in \mathscr{F}_{r}\left(\Gamma^{2}\right)$ be a 2-cocycle on $\Gamma(S, m)$ and $f \in \mathscr{F}_{r}\left(\Gamma^{1}\right)$, then the following are equivalent: 
(i) $\tau_{f} q \tilde{q}=1$,

(ii) $q=\tau_{h} \pi_{k}$ for some $h \in \mathscr{F}_{r}\left(\Gamma^{1}\right)$, where $k \tilde{k}=1$ and $f h \tilde{h}$ is multiplicative.

Proof. Suppose that $(q, f)$ satisfies (i). Then, by Proposition 2.3, $q=\tau_{h_{1}} \pi_{k_{1}}$ for some $h_{1} \in \mathscr{F}_{r}\left(\Gamma^{1}\right)$ and product cocycle $\pi_{k_{1}}$, moreover $k_{2}=\left(k_{1} \tilde{k}_{1}\right)^{-1}$ is both $\sim$-invariant and symmetric. Let $k_{3}$ be a measurable square root of $k_{2}$ which is also $\sim$-invariant and symmetric. Then $\pi_{k_{3}}=\tau_{g}$ for some $g \in \mathscr{F}_{r}\left(\Gamma^{1}\right)$, and if $k=k_{1} k_{3}, h=h_{1} g^{-1}$, then the pair $(h, k)$ satisfies (ii). The converse is immediate.

Proposition 4.4. Let $p$ be a multiplier on $\Gamma_{Z}$, then

$$
p(\omega, \alpha, \beta)=\varepsilon(\omega) \frac{g(\alpha \cup \tilde{\omega}) g(\omega \cup \beta)}{g(\alpha \cup \beta)} \frac{\pi(\alpha, \beta)}{\pi(\alpha, \tilde{\omega}) \pi(\omega, \beta)},
$$

where $\varepsilon \in \mathscr{F}_{r}\left(\Gamma^{1}\right)$ is multiplicative, $g \in \mathscr{F}_{r}\left(\Gamma^{1}\right)$ and $\pi=\pi_{k}$, where $k$ is determined by a skew-symmetric $\{ \pm i, 1\}$-valued function $\xi$ on $S_{0} \times S_{0}$, a skew-symmetric function $\gamma$ on $S_{+} \times S_{+}$and $a\{ \pm 1\}$-valued function $\eta$ on $S_{0} \times S_{+}$as follows:

$k(s, t)=\xi(s, t)$ on $S_{0} \times S_{0},=\left\{\begin{array}{lll}\gamma(s, t) & \text { on } & S_{+} \times S_{+} \\ \gamma(\tilde{s}, \tilde{t}) & S_{-} \times S_{-} \\ \gamma(\tilde{t}, s) & S_{+} \times S_{-} \\ \gamma(t, \tilde{s}) & S_{-} \times S_{+}\end{array}=\left\{\begin{array}{lll}\eta(s, t) & \text { on } & S_{0} \times S_{+} \\ \eta(t, \tilde{s}) & S_{-} \times S_{0} \\ 1 & S_{+} \times S_{0} \\ 1 & S_{0} \times S_{-}\end{array}\right.\right.$

Proof. Combining Proposition 4.2 and Lemma 4.3 we obtain the form (4.6) for $p$ in which $\varepsilon \in \mathscr{F}_{r}\left(\Gamma_{1}\right)$ is multiplicative and $\pi=\pi_{k_{1}}$, where $k_{1}$ satisfies

$$
\begin{aligned}
k_{1} \tilde{k}_{1} & =1, \\
\frac{k_{1}(s, t) k_{1}(s, \tilde{t})}{k_{1}(t, s) k_{1}(\tilde{t}, s)} & =1 .
\end{aligned}
$$

By the analysis of Sect. 3 we may assume that $k_{1}$ has the required form on $S_{0} \times S_{0}$, and by taking a measurable logarithm of $k_{1}$ on $S_{+} \times S_{+}$(and arguing as in the proof of Proposition 2.3) we may assume that $\gamma:=\left.k_{1}\right|_{s_{+} \times s_{+}}$has the required form also. Let $\zeta=\left.k_{1}\right|_{s_{+} \times s_{-}}, \delta=\left.k_{1}\right|_{s_{+} \times s_{0}}$ and $\alpha=\left.k_{1}\right|_{s_{0} \times S_{+}}$then by $(4.8 \mathrm{~b}) \eta:(s, t) \mapsto$ $\delta(t, s) \alpha(s, t)^{-1}$ is $\{ \pm 1\}$-valued on $S_{0} \times S_{+}$, thus $k_{1}$ has the following form on the rest of $S^{(2)}$ :

$$
k_{1}(s, t)=\left\{\begin{array}{lll}
\gamma(\tilde{s}, \tilde{t}) & \text { on } & S_{-} \times S_{-} \\
\zeta(t, s) \gamma(t, \tilde{s}) \gamma(\tilde{s}, t)^{-1} & & S_{-} \times S_{+} \\
\delta(\tilde{t}, s)^{-1} & & S_{0} \times S_{-} \\
\delta(t, s) \eta(s, t) & & S_{0} \times S_{+} \\
\delta(\tilde{s}, t)^{-1} \eta(t, \tilde{s}) & S_{-} \times S_{0}
\end{array}\right.
$$

Define $k_{2}$ on $S^{(2)}$ by

$$
k_{2}(s, t)=\left\{\begin{array}{lll}
\gamma(\tilde{t}, s) \zeta(s, t)^{-1} & \text { on } & S_{+} \times S_{-} \\
\gamma(\tilde{s}, t) \zeta(t, s)^{-1} & & S_{-} \times S_{+}
\end{array},=\left\{\begin{array}{lll}
\delta(s, t)^{-1} & \text { on } & S_{+} \times S_{0} \\
\delta(t, s)^{-1} & & S_{0} \times S_{+} \\
\delta(\tilde{t}, s) & S_{0} \times S_{-} \\
\delta(\tilde{s}, t) & S_{-} \times S_{0}
\end{array}\right.\right.
$$


and 1 elsewhere, then $k_{2}$ satisfies (4.8) and is symmetric, so $\pi_{k_{2}}$ is trivial. The product $k_{1} k_{2}$ is of the required form and the proof is complete.

We write $(f, g, k)$ for the multiplier (4.6) where $\varepsilon=\varepsilon_{f}$ and $\pi=\pi_{k}$. By taking a measurable logarithm of $f$ one may divide out the $\sim$-invariant part of $\varepsilon$ to obtain a representation $\left(f^{\prime}, g^{\prime}, k\right)$ in which $\tilde{f}^{\prime}=f^{\prime-1}$. Then the extent to which the representation of a given multiplier is unique is as follows: $(f, g, k) \equiv 1$ if and only if $k \equiv 1, g$ is multiplicative, $\varepsilon_{f}=g \tilde{g}$ and is $\{ \pm 1\}$-valued. As before we define equivalence for multipliers by equivalence of the corresponding 2-cocycles (modulo null sets), and denote the group of equivalence classes of multipliers on $\Gamma_{Z}$ by $\mathscr{M}\left(\Gamma_{Z}\right)$. We remind the reader of our convention that a group valued function $f$ on $Y \times Y$ is skew-symmetric if $f(t, s)=f(s, t)^{-1}$.

Lemma 4.5. With the above notation let $p$ and $p^{\prime}$ be multipliers on $\Gamma_{Z}$. Then $p \approx p^{\prime}$ if and only if almost everywhere $\xi / \xi^{\prime}$ and $\gamma / \gamma^{\prime}$ are $\{ \pm 1\}$-valued and $\eta=\eta^{\prime}$.

Proof. $p$ and $p^{\prime}$ are equivalent if and only if $\xi / \xi^{\prime}, \gamma / \gamma^{\prime}$ and $\eta / \eta^{\prime}$ are symmetric outside a null set. But $\xi / \xi^{\prime}$ and $\gamma / \gamma^{\prime}$ are skew-symmetric and $\eta / \eta^{\prime}(t, s)=\eta / \eta^{\prime}(s, t)$ if and only if $\eta / \eta^{\prime}(s, t)=\eta / \eta^{\prime}(t, s)=1$. The result follows.

Combining this with Proposition 4.4, identifying $\eta$ with its support $\eta^{-1}(\{-1\})$ and again accommodating the group isomorphism between $\mathbb{C}^{\times} /\{ \pm 1\}$ and $\mathbb{C}^{\times}$, we have:

\section{Theorem 4.6.}

$$
\mathscr{M}\left(\Gamma_{Z}\right) \cong\left(\mu\left(\Gamma_{2}\left(Z_{0}\right)\right), \Delta\right) \times \mathscr{A}_{r}\left(Z_{+}^{(2)} ; \mathbb{C}^{\times}\right) \times\left(\mu\left(Z_{0} \times Z_{+}\right), \Delta\right),
$$

where $\mathscr{A}_{r}$ is the group of (equivalence classes of) skew-symmetric functions with relatively compact range.

Finally notice that if $p=(f, g, k)$ and $p^{\prime}=\left(f^{\prime}, g^{\prime}, k^{\prime}\right)$ are equivalent multipliers on $\Gamma_{Z}$, then $\mathscr{M}_{h g / g^{\prime}}$ is an isomorphism from $\left(\mathscr{K}\left(S, m, i, S_{+}\right), \tilde{*}_{p}\right)$ to $\left(\mathscr{K}\left(S, m^{\prime}, i, S_{+}\right), \tilde{*}_{p}\right)$, where $m^{\prime}$ is the measure given by $d m^{\prime}=\left(f / f^{\prime}\right) d m$, if $h$ is the almost everywhere $\{ \pm 1\}$-valued function $\sigma \rightarrow \prod_{\{s, t\}=\sigma}\left(k^{\prime} / k\right)(s, t)$.

\section{Levy Fields and Bose-Fermi Bridges}

In this section $\mathrm{X}=(S, m)$ is a fixed (non-atomic, $\sigma$-finite) measure space, so we abbreviate $\mathscr{K}(X)$ to $\mathscr{K}, d m(s)$ to $d s$ and so on. $L^{2}(X)$ is naturally included in $L^{2}(\Gamma)$, in fact in $\mathscr{K}$-we use this without further comment.

A field of operators $\left\{l_{q}(f): f \in \mathscr{K}\right\}$, with common domain $\mathscr{K}$, is associated with each bounded 2-cocycle $q$. It is shown that $\delta_{\varnothing}$ is cyclic for $\left\{l_{q}(f): f \in L^{2}(X)\right\}$ and that $\left\{l_{q}(f), l_{q}^{\dagger}(f): f \in L^{2}(X)\right\}$ is irreducible. Local commutation relations are then obtained under various conditions to be satisfied by the cocycles on sets in $\Gamma^{(2)}$ determined by the supports of the test functions. In particular we obtain bridges from Boson to Fermion quantum fields, and also a continuum of inequivalent smooth Levy fields with common covariance. This highlights some of the obstacles in the problem of deducing commutation relations from martingale conditions which was raised in [AP]. 
Definition 5.1. Define a map ^ from $F\left(\Gamma^{(2)}\right)$ to operators on $F(\Gamma)$, a product $\times$ on $F\left(\Gamma^{(2)}\right)$ and a bilinear map $F(\Gamma) \times F\left(\Gamma^{(2)}\right)$ to $F\left(\Gamma^{(2)}\right)$ by

$$
\begin{aligned}
\hat{v} g(\sigma) & =\sum_{|\alpha|=\sigma} v\left(\alpha_{1}, \alpha_{2}\right) g\left(\alpha_{2}\right), \\
u \times v(\sigma, \tau) & =\sum_{|\alpha|=\sigma} u\left(\alpha_{1}, \alpha_{2} \cup \tau\right) v\left(\alpha_{2}, \tau\right), \\
g . v(\sigma, \tau) & =g(\sigma) v(\sigma, \tau) .
\end{aligned}
$$

The map ^ is well defined by (3.6) and is clearly injective so that associativity for the product $\times$ follows from the easily verified relation $\widehat{u \times v}=\hat{u} \hat{v}$. If $u=g \cdot v$ where $g \in \mathscr{K}$ and $v$ is bounded then, by (3.6), $\hat{u}$ leaves $\mathscr{K}$ invariant permitting the following

Definition 5.2. For a bounded 2-cocycle $q$ and function $f$ in $\mathscr{K}$, define an operator on $L^{2}(\Gamma)$ with domain $\mathscr{K}$ by

$$
l_{q}(f)=\widehat{f . q .}
$$

By the previous remarks each operator leaves $\mathscr{K}$ invariant and if $\left(q_{i}, f_{i}\right) i=1, \ldots, n$ are as above

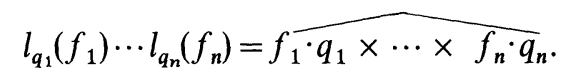

Moreover

$$
l_{q_{1}}\left(f_{1}\right) \cdots l_{q_{n}}\left(f_{n}\right) f_{n+1}=f_{1} \circ_{q_{1}} f_{2}{ }^{\circ} q_{2} \cdots \circ_{q_{n}} f_{n+1},
$$

where the right-hand side is independent of bracketing, generalising the associativity of $\circ_{q}(2.8)$.

Lemma 5.3. Let $f \in \mathscr{K}$ and let $q$ be a bounded 2-cocycle. Then $\mathscr{K} \subset \mathscr{D}\left(l_{q}(f)^{*}\right)$ and

$$
\left[l_{q}^{\dagger}(f) g\right](\sigma)=\int \bar{f}(\omega) \bar{q}(\omega, \sigma) g(\sigma \cup \omega) d \omega
$$

where $l_{q}^{\dagger}(f)$ denotes the restriction of $l_{q}(f)^{*}$ to $\mathscr{K}$.

Proof. For $g \in \mathscr{K}$ the integral (5.6) is well defined, and the identity is established by taking inner products and applying the integral-sum lemma.

Note. For operators $c$ having domain $\mathscr{K}$ we shall consistently use the notation $c^{\dagger}$ to denote the restriction to $\mathscr{K}$ of the adjoint of $c$. Moreover a sentence or equation involving terms $c^{\#}$ is to be understood as two statements: one in which each \# is replaced by $\dagger$ and another in which each \# is deleted. Elements of $\mathscr{K}$ will sometimes be referred to as test functions, and those whose support is contained in $\Gamma_{1}, \Gamma_{n}$ or $\Gamma_{0} \cup \cdots \cup \Gamma_{m}$ (for some $m$ ) as 1-particle, $n$-particle or finite particle functions respectively.

Proposition 5.4. Let $q_{1}, \ldots, q_{n}$ be bounded 2-cocycles and $f_{1}, \ldots, f_{n}$ belong to $L^{2}(X)$, then

$$
l_{q_{1}}\left(f_{1}\right) \cdots l_{q_{n}}\left(f_{n}\right) \delta_{\varnothing}=n ! P_{S} Q_{n} f_{1} \otimes \cdots \otimes f_{n},
$$

where $Q_{n}(\mathbf{s})=\prod_{i=1}^{n-1} q_{i}\left(s_{i} ; s_{i+1}, \ldots, s_{n}\right), P_{S}$ is the symmetrisation projection: $(n !)^{-1} \sum_{\pi \in \mathscr{S}_{n}} U_{\pi}$ $\left(\left(U_{\pi} f\right)(\mathbf{s})=f\left(s_{\pi^{-1}(1)}, \ldots, S_{\pi^{-1}(n)}\right)\right)$ and the left-hand side is considered as a symmetric function on $S^{(n)}$. 
In particular for any sequence $\left\{q_{i}: i \in \mathbb{N}\right\}$ of bounded 2-cocycles the linear span of

$$
\left\{l_{q_{1}}\left(f_{1}\right) \cdots l_{q_{n}}\left(f_{n}\right) \delta_{\varnothing}: n \in \mathbb{N}, f_{1}, \ldots, f_{n} \in L^{2}(X)\right\}
$$

is dense in $L^{2}(\Gamma)$.

Proof. A straightforward induction gives the first part and the second part follows from the fact that, for each $n,\left\{Q_{n} f_{1} \otimes \cdots \otimes f_{n}: f_{i} \in L^{2}(X)\right\}$ spans $L^{2}\left(X^{n}\right)$ since each $Q_{n}$ is non-vanishing.

Proposition 5.5. Let $q$ be a bounded 2-cocycle, then

$$
\left\{l_{q}(f), l_{q}^{\dagger}(f): f \in L^{2}(X)\right\}
$$

is irreducible in the sense that the only bounded operators which, together with their adjoint, leave $\mathscr{K}$ invariant and commute with this set are the multiples of the identity. Proof. Suppose that $T$ is an operator on $L^{2}(\Gamma)$ which commutes with the family (5.8) and, together with its adjoint, leaves $\mathscr{K}$ invariant. Then, since (5.8) is symmetric, $T^{\dagger}$ will commute with this class, and

$$
T\left(f_{1} \circ f_{2}\right)=T l\left(f_{1}\right) f_{2}=f_{1} \circ T f_{2} ; \quad T^{\dagger}\left(f_{1} \circ f_{2}\right)=f_{1} \circ T^{\dagger} f_{2} .
$$

Letting $h=T \delta_{\varnothing}, k=T^{\dagger} \delta_{\varnothing}$ and iterating (5.9) we have

$$
T f=f \circ h ; \quad T^{\dagger} f=f \circ k
$$

for $f$ in $\mathscr{K}_{0}:=$ linear $\operatorname{span}\left\{f_{1} \circ \cdots \circ f_{n}: n \in \mathbb{N}, f_{i} \in L^{2}(X)\right\}$. Thus for $f \in \mathscr{K}_{0}$,

$$
\left\langle\delta_{\varnothing}, T^{*} g\right\rangle=\langle h, g\rangle=\int \bar{h}(\sigma) g(\sigma) d \sigma
$$

whereas

$$
\left\langle\delta_{\varnothing}, T^{\dagger} g\right\rangle=\left\langle\delta_{\varnothing}, g \circ k\right\rangle=g(\varnothing) k(\varnothing),
$$

so that $h=k(\varnothing) \delta_{\varnothing}$. In other words $T$ is a multiple of the identity on $\mathscr{K}_{0}$ which, by the previous proposition, is dense in $L^{2}(\Gamma)$. Thus if $T$ is bounded then it must be a multiple of the identity.

Proposition. 5.6. Let $f, g \in \mathscr{K}$ and $\xi, \eta$ be bounded product cocycles, then for $\varepsilon, \delta \in \mathbb{C}$,

$$
l_{\eta}(g) l_{\xi}(f)+\delta l_{\xi}(f) l_{\eta}(g)=\hat{q}
$$

where

$$
q(\sigma, \tau)=\sum_{\alpha \subset \sigma} g(\alpha) f(\bar{\alpha}) \eta(\alpha, \tau) \xi(\bar{\alpha}, \tau)\{\eta(\alpha, \bar{\alpha})+\delta \xi(\bar{\alpha}, \alpha)\}
$$

and if $f$ and $g$ are one-particle functions, then

$$
\begin{aligned}
& \left(\left\{l_{\eta}^{\dagger}(g) l_{\xi}(f)+\varepsilon l_{\xi}(f) l_{\eta}^{\dagger}(g)\right\} h\right)(\sigma) \\
& \quad=\Omega_{f, g ; \xi, \eta}(\sigma) h(\sigma)+\int \sum_{s \in \sigma} f(s) \bar{g}(t) h(\{t\} \cup \sigma \backslash\{s\}) \frac{\xi(s ; \sigma) \bar{\eta}(t ; \sigma)}{\bar{\eta}(t ; s)}\{\xi(s ; t) \bar{\eta}(t ; s)+\varepsilon\} d t,
\end{aligned}
$$

where $\Omega(\sigma)=\int \bar{g} f(t) \bar{\eta} \xi(t, \sigma) d t$.

Proof. Remembering our convention that cocycles take the value 1 on the set diagonals, these identities are immediate. 
For $\varepsilon \in \mathbb{C}$, define a bracket on the algebra of operators on $\mathscr{K}$ by

$$
[X, Y]_{\varepsilon}=X Y+\varepsilon Y X .
$$

For $\varepsilon= \pm 1$ this is the anti-commutator (Jordan product) and commutator (Lie bracket) respectively - in general it is a deformation of these.

Corollary 5.7. Let $f, g \in \mathscr{K}$ and let $\xi, \eta$ be bounded product cocycles, then for $\delta, \varepsilon \in \mathbb{C}$,

(i) if $\tilde{\eta}+\delta \xi=0$ on $\operatorname{supp} f \times \operatorname{supp} g$, then $\left[l_{\eta}(f), l_{\xi}(g)\right]_{\delta}=0$,

(ii) if $f$ and $g$ are one-particle functions and $\xi \tilde{\bar{\eta}}+\varepsilon=0$ on $\operatorname{supp} f \times \operatorname{supp} g$, then $\left[l_{\eta}^{\dagger}(g), l_{\xi}(f)\right]_{\varepsilon}=M_{\Omega_{f, g ; \xi, \eta}}$, the multiplication operator.

In particular, if $f$ is a one-particle function and $\eta \tilde{\bar{\eta}}+\varepsilon=0$ on $\operatorname{supp} f \times \operatorname{supp} f$ where $\varepsilon>0$, then $l_{n}(f)$ is bounded.

Corollary 5.8. Let $q$ be the product cocycle determined by a function of the form $k_{F}$ (defined in (3.18)) and let $E=F \cup \tilde{F}$, then for $f, g \in L^{2}(X)$,

(i) $\operatorname{supp} f \times \operatorname{supp} g \subset\left\{\begin{array}{l}E \\ E^{c}=S \backslash E\end{array} \Rightarrow[l(f), l(g)]_{ \pm}=0 ;\left[l^{\dagger}(g), l(f)\right]_{ \pm}=\langle g, f\rangle\right.$,

(ii) If supp $f \subset \sum_{j=1}^{n} A_{j}$ for a finite collection of measurable subsets $\left\{A_{j}\right\}$ of $S$ for which $\bigcup_{j=1}^{n} A_{j} \times A_{j} \subset E$, then $l_{q}(f)$ is bounded.

Proof. On $E, q+\tilde{q}=0$ and $q \tilde{\bar{q}}+1=0$, whereas on $E^{c}, q=\bar{q}=\tilde{q}=1$. The result follows from the linearity of $l_{q}(\cdot)$ :

$$
l_{q}(f)=\sum_{j} l_{q}\left(f \chi_{A_{j}}\right) \text {, each } l_{q}\left(f \chi_{A_{j}}\right) \text { is bounded. }
$$

An interesting class of examples where the above applies is given by $X=\left(\mathbb{R}^{k}\right.$, Lebesgue) and $F$ such that $E=\{(x, y):|x-y|<\lambda\}, \lambda \in(0, \infty)$, then $l(f)$ is bounded whenever the test function $f$ has compact support. We shall return to this example shortly.

Right o-multiplication may be similarly defined:

$$
r_{q}(f) g=g^{\circ} f \quad(f, g \in \mathscr{K}) .
$$

By the generalised associativity (5.5), the left and right o-multiplication operators commute. When the cocycle takes particular form we can say more:

Proposition 5.9. Again let $q$ be the product cocycle determined by a function of the form $k_{F}$, then there is a $\{ \pm 1\}$-valued function $\psi$ on $\Gamma$ such that

$$
M_{\psi} l(f) M_{\psi}^{-1}=r(\psi f), \quad f \in \mathscr{K} .
$$

Proof. Let $f, g \in \mathscr{K}$, then

$$
[r(f) g](\sigma)=\sum_{\alpha \subset \sigma} q(\bar{\alpha}, \alpha) f(\alpha) g(\bar{\alpha})=\sum_{\alpha \subset \sigma} q(\alpha, \bar{\alpha}) q^{2}(\bar{\alpha}, \alpha) f(\alpha) g(\bar{\alpha}),
$$

but $q^{2}$ is skew-symmetric and $\{ \pm 1\}$-valued-it is therefore symmetric and so equal to $\tau_{\psi}$ for some $\{ \pm 1\}$-valued function $\psi$. Since $\psi=\psi^{-1}(5.10)$ follows. 
For each multiplier $p$ on $\Gamma$ and test function $f$ in $\mathscr{K}$, let $L_{p}(f), R_{p}(f)$ be the left and right $*$-multiplication operators on $\mathscr{K}$ :

$$
L_{p}(f): g \rightarrow f *_{p} g ; \quad R_{p}(f): g \rightarrow g *_{p} f .
$$

Proposition 5.10. Let $p$ be a multiplier on $\Gamma, f, g \in \mathscr{K}$.

(i) $L_{p}^{\dagger}(f) g=\bar{f} *_{\hat{p}} g$, where $\hat{p}:(\omega, \alpha, \beta) \rightarrow \bar{p}(\alpha, \omega, \beta)$.

(ii) If $p(\omega, \alpha, \beta)=\eta(\alpha, \beta) / \eta(\alpha, \omega) \eta(\omega, \beta)$, where $\eta$ is a skew-symmetric, unit modulus 2-cocycle, then

$$
L_{p}^{\dagger}(f)=L_{p}(\bar{f}) .
$$

(iii) If further $f$ is a one-particle function, then

$$
\begin{aligned}
& L_{p}(f)=l_{\eta}(f)+l_{\eta}^{\dagger}(\bar{f}), \\
& R_{p}(f)=l_{\tilde{\eta}}(f)+l_{\tilde{\eta}}^{\dagger}(\bar{f})=L_{\tilde{p}}(f),
\end{aligned}
$$

where

Proof.

$$
\tilde{p}:(\omega, \alpha, \beta) \rightarrow p(\omega, \beta, \alpha)
$$

(i) Follows from the applications of the integral-sum lemma and (ii) and (iii) are immediate.

Proposition 5.11. Let $f, g, h \in \mathscr{K}$ and $\xi, \eta$ be bounded 2-cocycles, then

$$
\begin{aligned}
& \left\|\left[l_{\xi}^{\dagger}(f)-l_{\eta}^{\dagger}(g)\right] h\right\| \leqq\left\|\sqrt{3^{N}} h\right\|\left\{\|f\|\|\xi-\eta\|_{\infty}+\|\eta\|_{\infty}\|f-g\|\right\}, \\
& \left\|\left[l_{\xi}(f)-l_{\eta}(g)\right] h\right\| \leqq\left\|\sqrt{3}^{N} h\right\|\left\{\left\|\sqrt{3}^{N} f\right\|\|\xi-\eta\|_{\infty}+\|\eta\|_{\infty}\left\|\sqrt{3^{N}}(f-g)\right\|\right\} .
\end{aligned}
$$

Proof. Several applications of the integral-sum lemma give the identities

$$
\begin{aligned}
\left\langle h, l_{\xi}(f) l_{\eta}^{\dagger}(g) h\right\rangle= & \iiint \bar{h}(\alpha \cup \beta) f(\alpha) \xi(\alpha, \beta) \bar{g}(\gamma) \bar{\eta}(\gamma, \beta) h(\beta \cup \gamma) d \alpha d \beta d \gamma, \\
\left\langle h, l_{\xi}^{\dagger}(f) l_{\eta}(g) h\right\rangle= & \iiint \int \bar{h}(\alpha \cup \beta) \bar{f}(\gamma \cup \delta) \bar{\xi}(\gamma \cup \delta, \alpha \cup \beta) \eta(\alpha \cup \gamma, \beta \cup \delta) \\
& \cdot g(\alpha \cup \gamma) h(\beta \cup \delta) d \alpha d \beta d \gamma d \delta .
\end{aligned}
$$

Applying the estimate $\int\left\|k^{\alpha}\right\|^{2} d \alpha \leqq\left\|\sqrt{3}^{N} k\right\|^{2}(k \in \mathscr{K})$, which follows from (3.7), then gives the inequalities (5.11).

Corollary 5.12. Let $\xi=\pi_{k_{1}}, \eta=\pi_{k_{2}}$ be bounded product cocycles and $f, g$ be oneparticle functions. For $h$ in the linear span of the exponential vectors $\left\{\varepsilon_{f}: f \in L^{2}(X)\right\}$,

$$
\max \left\{\left\|\left[l_{\xi}(f)-l_{\eta}(g)\right] h\right\|,\left\|\left[l_{\xi}^{\dagger}(f)-l_{\eta}^{\dagger}(g)\right] h\right\|\right\} \leqq c\left\{\|f\|\left\|k_{1}-k_{2}\right\|_{\infty}+\|f-g\|\right\},
$$

where $c$ is a constant dependent only on $h$.

Let $F \subset S^{(2)}$ be such that $F \cup \tilde{F}$ is a measurable partition of $S^{(2)}$ and consider the family of 2-cocycles $\left\{\xi_{v}: v \in[0,1]\right\}$, where $\xi_{v}$ is the product cocycle determined by the function $k:=e^{i v \pi / 2} \chi_{F}+e^{-i v \pi / 2} \chi_{\tilde{F}}$, then $\left\|\xi_{v}-\xi_{\psi}\right\|<\left((\pi / 2) e^{\pi / 2}\right)|v-\psi|$, so $\left\{l^{\nu}: f \mapsto l_{\xi_{v}}(f) \mid v \in[0,1]\right\}$ is a homotopy between the boson and fermion Fock representations over $L^{2}(X)$ - the first Bose-Fermi bridge.

In the notation of (3.7) the following estimates are easily obtained by more applications of the integral-sum identity. 
Proposition 5.13. Let $f, g, h \in \mathscr{K}$ and let $\xi, \eta$ be bounded 2-cocycles, then

$$
\begin{aligned}
K(\xi, \eta)^{-1}\left\|\left[l_{\xi}^{\dagger}(f)-l_{\eta}^{\dagger}(g)\right] h\right\|^{2} \leqq & \iint_{E_{1}(f, \xi, \eta)}|f(\alpha)|\left\|h^{\alpha}\right\||f(\beta)|\left\|h^{\beta}\right\| d \alpha d \beta \\
& +c(f, g, h)\|f-g\|, \\
K(\xi, \eta)^{-1}\left\|\left[l_{\xi}(f)-l_{\eta}(g)\right] h\right\|^{2} \leqq & \iint_{E_{2}(f, \xi, \eta)}\left\|f^{\alpha}\right\|\left\|h^{\alpha}\right\|\left\|f^{\beta}\right\|\left\|h^{\beta}\right\| d \alpha d \beta \\
& +c\left(\sqrt{3^{N}} f, \sqrt{3}^{N} g, h\right)\left\|\sqrt{3}^{N}(f-g)\right\|,
\end{aligned}
$$

where

$$
\begin{aligned}
& E_{1}=\{(\alpha, \beta) \in \operatorname{supp} f \times \operatorname{supp} f:(\xi-\eta)(\alpha),(\xi-\eta)(\beta) \not \equiv 0 \text { as functions of } \gamma\}, \\
& E_{2}=S_{f} \times S_{f} \cap S_{\xi-\eta} \cap S_{\xi-\eta}, \\
& c(f, g, h)=\left\|\sqrt{3}^{N} h\right\|^{2}\{\|f-g\|+2\|f\|\} ; \quad K(\xi, \eta)=5 \max \left\{\|\xi\|_{\infty}^{2}\|\eta\|_{\infty}^{2}\right\},
\end{aligned}
$$

and $S_{q}=\{(\alpha, \beta): \alpha \subset \sigma, \beta \subset \tau, q(\sigma, \tau)=0\}$ with similar definition for one argument functions.

Let $X$ be a finite dimensional Euclidean space with Lebesgue measure and $\left\{\eta_{\lambda}: \lambda \in \mathbb{R}_{+}\right\}$be the product cocycles determined by functions $k_{F_{\lambda}}$ (see 3.18) $\left(F_{\lambda}=\left\{(s, t):|s-t|<\lambda, s_{1}<t_{1}\right\}\right.$, then for functions $f$ of compact support the sets $E_{i}\left(f, \eta_{\lambda}, \eta_{\mu}\right)(i=1,2)$ tend to zero, as $\lambda$ approaches $\mu$, in measure. Since, for $\lambda=0$, $l_{\lambda}^{\#}: f \mapsto l_{\lambda}^{\#}(f)$ is the boson Fock representation and as $\lambda \rightarrow \infty, l_{\lambda}^{\#}$ approaches the Fermion Fock representation, we have a second Bose-Fermi bridge. In fact, if $f$ and $g$ are one particle functions and $\{|s-t|: f(s), g(t) \neq 0\} \subset[0, \lambda]$, then $l_{\lambda}^{\#}(f), l_{\lambda}^{\#}(g)$ satisfy the canonical anti-commutation relations (Corollary 6.8) whereas if $\{|s-t|: f(s), g(t) \neq 0\} \subset(\lambda, \infty)$ they satisfy the canonical commutation relations. If $f$ and $g$ have compact support then the former condition will eventually be satisfied (for large enough $\lambda$ ).

Quasi-free, or positive temperature, Bose-Fermi bridges may also be constructed; either via fields of operators $\left\{L_{p}(f): f \in L^{2}(S, m)\right\}$ defined through suitable multipliers $p$ on a twisted finite power set $\Gamma_{Z}$, or by employing linear/conjugate linear operators $R, T$ on $L^{2}(S, m)$ and defining

$$
L(f)=l_{\xi}(R f)+l_{\eta}^{\dagger}(T f) \quad f \in L^{2}(S, m)
$$

for well-chosen pairs of 2-cocycles $(\xi, \eta)$.

The central result of [A P] (see also [P]) is that two smooth Levy boson (fermion) fields with a given covariance kernel are equivalent, and that smooth Levy boson (fermion) fields may be constructed for any covariance density. The question of whether other smooth Levy fields exist or not is left open but has been a source of lively speculation. We now demonstrate the existence of a plentiful supply of such smooth Levy fields in which the past and future satisfy no fixed (anti-) commutation relations. A Levy-type theorem characterising quantum Brownian motion thus remains beyond our reach at present. The reader is referred to [A P] for the definitions of Levy-field, covariance kernel etc.

Lemma 5.14. Let $\xi$ be a bounded 2-cocycle and let $f, g, h \in \mathscr{K}$ be such that supp $g \subset \Gamma_{I}$ and supp $g \cup \operatorname{supp} h \subset \Gamma_{J}$, where $I \cap J=\varnothing$, then 


$$
\left\langle f,\left[l_{\xi}^{\#}(g)-g^{\#}(\varnothing)\right] h\right\rangle=0,
$$

where $g^{\dagger}:=\bar{g}$.

Proof. Under the above asumptions on $f, g, h$ and $\xi$,

$$
\begin{aligned}
\left\langle f, l_{\xi}(g) h\right\rangle & =\int \bar{f}(\sigma) \sum_{\alpha \subset \sigma} \xi(\alpha, \bar{\alpha}) g(\alpha) h(\bar{\alpha}) d \sigma \\
& =\iint \bar{f}(\alpha \cup \beta) \xi(\alpha, \beta) g(\alpha) h(\beta) d \alpha d \beta=g(\varnothing)\langle f, h\rangle
\end{aligned}
$$

by the integral sum identity and (5.13) follows.

Proposition 5.15. Let $q$ be a unit modulus 2-cocycle on $\Gamma\left(\mathbb{R}_{+}\right)$, then the family

$$
\mathfrak{X}=\left\{X(f):=l_{q}(f)+l_{q}^{\dagger}(f) \mid f \in L^{2}\left(\mathbb{R}_{+}\right)\right\}
$$

is a smooth Levy field, with cyclic vector $\delta_{\varnothing}$ and covariance kernel $(f, g) \rightarrow \int \bar{f} g$, whenever $\mathfrak{X}$ consists of bounded operators.

Proof. Since $l_{q}(f)=\frac{1}{2}\{X(f)-i X(i f)\}, \delta_{\varnothing}$ is cyclic for the linear span of $\left\{X\left(f_{1}\right) \ldots\right.$ $\left.X\left(f_{n}\right) \delta_{\varnothing}: n \in \mathbb{N}, f_{i} \in L^{2}\left(\mathbb{R}_{+}\right), i=1, \ldots, n\right\}$ by Proposition 5.4. $\left(f_{1}, \ldots, f_{n}\right) \mapsto X\left(f_{n}\right) \delta_{\varnothing}$ is clearly real multilinear, and for fixed $f^{(1)}, \ldots, f^{(n)}$ the continuity of the map $t \mapsto X\left(f_{t]}^{(1)}\right) \cdots X\left(f_{t]}^{(n)}\right) \delta_{\varnothing}$ is ensured by the estimates (5.11). The martingale property: $\left\langle u, X\left(f_{[t}\right) v\right\rangle=0$ whenever supp $u, v \subset \Gamma_{[0, t]}$, is an instance of Lemma 5.14; in fact $l_{q}^{\dagger}\left(f_{[t}\right) u=l_{q}^{\dagger}\left(f_{[t}\right) v=0$ and

$$
\begin{aligned}
\left\langle u, X\left(f_{[t}\right) X\left(g_{[t}\right) v\right\rangle & =\left\langle u, l_{q}^{\dagger}\left(f_{[t}\right) l_{q}\left(g_{[t}\right) v\right\rangle \\
& =\int_{[0, t]} \int_{t}^{\infty} d \beta d s \bar{u}(\beta) \bar{f}(s) g(s) v(\beta)=\langle u, v\rangle \int_{t}^{\infty} \bar{f} g
\end{aligned}
$$

in other words $\mathfrak{X}$ is a Levy field with the given cyclic vector and covariance kernel. Now suppose that $u \in L^{2}(\Gamma)$ has support in $\Gamma_{[0, s]}$ and $f, g \in L^{2}\left(\mathbb{R}_{+}\right)$have support in $[s, t]$, then it is easy to see that $l^{\dagger}(f) l(g) u=\langle f, g\rangle u$, so that

$$
\begin{aligned}
\|\{X(f) X(g)-\langle f, g\rangle\} u\|^{2} & =\|l(f \circ g) u\|^{2} \\
& =\iint d \beta d \gamma \bar{u}(\beta) \overline{f \circ g}(\gamma) f \circ g(\gamma) u(\beta) \\
& =\|u\|^{2}\|f \circ g\|^{2} \leqq 4\|u\|^{2}\|f \otimes g\|^{2} .
\end{aligned}
$$

The smoothness condition now follows from this inequality by the dominated convergence theorem.

Theorem 5.16. For $\lambda \geqq 0$ let $\mathfrak{X}^{\lambda}$ be the Levy field (5.14) in which the 2-cocycle is the (skew-symmetric) product cocycle determined by the function

$$
(s, t) \rightarrow\left\{\begin{aligned}
i & \text { on } F_{\lambda} ; \\
-i & \text { on } \tilde{F}_{\lambda} ; \\
l & \text { elsewhere }
\end{aligned}\right.
$$

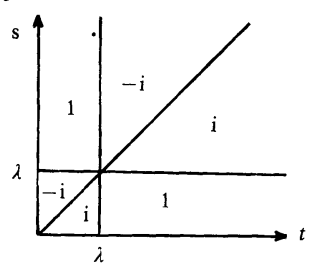

where $F_{\lambda}=\{(s, t): s<t<\lambda$ or $\lambda<s<t\}$. Then the family $\left\{\mathfrak{X}^{\lambda}: \lambda \geqq 0\right\}$ consists of inequivalent smooth Levy fields with common covariance kernel $(f, g) \rightarrow \int \bar{f} g$. 
Proof. By Corollary 5.8 (ii) $\mathfrak{X}^{\lambda}$ consists of bounded operators for each $\lambda\left(\mathbb{R}_{+}=\right.$ $\left.[0, \lambda) \cup[\lambda, \infty),(0, \lambda) \times[0, \lambda) \cup[\lambda, \infty) \times[\lambda, \infty)=F_{\lambda} \cup \tilde{F}_{\lambda}\right)$. Since these fields satisfy distinct local commutation relations, they must be inequivalent.

Remark. The Levy field $\mathfrak{X}^{0}$ is equivalent to the Levy fermion field. Smooth Levy fields with more general covariance kernels (inequivalent to the Bose and Fermi fields) may be constructed from operators $l_{q}(f)$, where $q$ is a 2-cocycle on $\Gamma\left(S, m, i, S_{+}\right)$in which $S_{+}=J \times\{0\}, S_{0}=I, S_{-}=\{0\} \times J$, where $I \cup J$ is a partition of $\mathbb{R}_{+}, i$ interchanges $(s, 0)$ and $(0, s)$ and $m_{0}, m_{+}, m_{-}$are absolutely continuous measures on the line.

\section{Differential Equations with Mixed Noise}

In the final section we solve linear stochastic differential equations driven by mixed noise, giving the action of solutions on vectors from $\mathscr{K}$ explicitly. This extends results from [H P 1,2, Maa, ApH, Me 1 and L M 2]. Our formula (6.2) for the solution is inspired by Theorem 5.3 of [Maa]. The equations we treat are those having the form

$$
\begin{aligned}
d X_{t}= & \left\{\sum_{i} L_{i}(t) \Phi\left(\phi^{(i)}(\cdot, t)\right) d A_{i}^{*}(t)+\sum_{i, j} M_{i j}(t) \Phi\left(\lambda^{(i, j)}(\cdot, t)\right) d \Lambda_{i j}(t)\right. \\
& \left.+\sum_{j} N_{j}(t) \Phi\left(\psi^{(j)}(\cdot, t)\right) d A_{j}(t)+K(t) d t\right\} X_{t}
\end{aligned}
$$

on $h_{0} \otimes L^{2}\left(\Gamma\left(\mathbb{R}_{+}\right)^{n}\right)=L^{2}\left(\Gamma\left(\mathbb{R}_{+}\right)^{n} ; h_{0}\right)$. The reader is referred to [HP 1] for the definitions of the creation, gauge and annihilation processes: $A_{i}^{*}, \Lambda_{i j}$ and $A_{j}$ respectively, and for the meaning of Eq. (6.1) and a solution for (6.1). For a linear operator $T$ on $\oplus^{(n)} L^{2}\left(\mathbb{R}_{+}\right), \Phi(T)$ denotes the operator defined on $\mathscr{E}:=$ linear span $\left\{v \otimes \varepsilon_{\mathrm{f}}: v \in h_{0}, \mathbf{f} \in \Theta^{(n)} L^{2}\left(\mathbb{R}_{+}\right)\right\}$by $\Phi(T) v \otimes \varepsilon_{\mathrm{f}}=v \otimes \varepsilon_{T \mathrm{r}} . L_{i}, M_{i j}, N_{j}$ and $K$ are locally bounded, strongly measurable $\mathscr{B}\left(h_{0}\right)$-valued maps on $\mathbb{R}_{+}$and $\phi^{(i)}, \lambda^{(i, j)}$ and $\psi^{(j)}$ are locally bounded, measurable diagonal $M_{n}(\mathbb{C})$-valued maps on $\mathbb{R}_{+}^{2}$ which are adapted in the sense that they take the value $I$ on $\{(s, t): s>t\}$. Here $\mathscr{B}\left(h_{0}\right)$ is the algebra of bounded linear operators on the complex separable Hilbert space $h_{0}, M_{n}(\mathbb{C})$ is the algebra of $n \times n$ complex matrices and the indices $i, j$ run from 1 to $n$.

To state the theorem we need a little more notation. $\boldsymbol{\beta}=\left(\beta_{i}^{j}\right)$ will denote a matrix of elements of $\Gamma\left(\mathbb{R}_{+}\right)$, while $\boldsymbol{\beta}$ will denote the $n$-tuple of sets whose $i^{\text {th }}$ component is $\beta_{i}^{1} \cup \beta_{i}^{2} \cup \cdots \cup \beta_{i}^{n}$ with $\boldsymbol{\beta}$. similarly defined. For $n$-tuples of sets $\boldsymbol{\alpha}, \gamma$, the $n$-tuple whose $i^{\text {th }}$ component is $\alpha_{i} \cup \gamma_{i}$ will be denoted $\alpha \cup \gamma$ and $d \mu^{n}(\omega)$ will be abbreviated to $d \omega$.

Theorem 6.1. The quantum stochastic differential equation (6.1) has a unique solution, with initial condition $X_{0}=I$, given by: for $t \in \mathbb{R}_{+},|\sigma| \subset[0, t]$ and $k \in \mathscr{E}$ with support in $\Gamma([0, t])^{n}$,

$$
\left(X_{t} k\right)(\sigma)=\int \sum_{\alpha \cup \beta \cup \gamma=\sigma} P(\gamma, \alpha, \underset{\sim}{\beta}, \omega) x_{t}(\alpha, \underset{\sim}{\beta}, \boldsymbol{\omega}) k(\boldsymbol{\omega} \cup \boldsymbol{\beta} . \cup \gamma) d \omega,
$$

where, if $|(\alpha, \underset{\sim}{\beta}, \gamma)|=\left\{t_{1}<t_{2}<\cdots<t_{N}\right\}$, the kernel $x_{t}$ is given by

$$
\begin{aligned}
x_{t}(\alpha, \underset{\alpha}{\beta}, \gamma) & =P(\boldsymbol{\alpha} \cup \boldsymbol{\beta}, \alpha, \underset{\beta}{\beta}, \gamma) r_{t}(\boldsymbol{\alpha}, \underset{\sim}{\beta}, \gamma), \\
r_{t}(\alpha, \underset{\sim}{\beta}, \gamma) & =R_{t}\left(R_{t_{N}}^{-1} G\left(t_{N}\right) R_{t_{N}}\right) \cdots\left(R_{t_{1}}^{-1} G\left(t_{1}\right) R_{t_{1}}\right),
\end{aligned}
$$


$R$ being the solution of the ordinary differential equation

$$
\frac{d R}{d t}=K R ; \quad R_{0}=I
$$

$G$ being given by

$$
G(t)=\left\{\begin{array}{lll}
L_{i}(t) & \text { if } & t \in \alpha_{i} \\
M_{i j}(t) & \text { if } & t \in \beta_{i}^{j} \\
N_{j}(t) & \text { if } & t \in \gamma_{j}
\end{array}\right.
$$

and the multiplier $P$ being given by

$$
\begin{aligned}
P(\omega, \alpha, \underset{\sim}{\beta}, \gamma) & =P_{1}(\omega, \alpha) P_{2}(\omega, \underset{\sim}{\beta}) P_{3}(\omega, \gamma) \\
& =\prod_{1 \leqq i, j, k \leqq n} \prod_{\substack{a \in \alpha_{i}, b \in \beta_{i}^{J} \\
c \in \gamma_{j}, t \in \omega_{k}}} \phi_{k}^{(i)}(t, a) \lambda_{k}^{(i, j)}(t, b) \psi_{k}^{(j)}(t, c) .
\end{aligned}
$$

Proof (sketch). In the above notation consider the family of functions $\mathscr{F}=$ $\left\{F(u, v ; f, g, \cdot): u, v \in h_{0}, \mathbf{f}, \mathbf{g} \in \oplus^{(n)} L^{2}\left(\mathbb{R}_{+}\right)\right\}$given by

$$
t \mapsto \int_{\Gamma([0, t])^{n}} \sum_{\alpha \cup \beta \cup \gamma=\omega}\left\langle V(\alpha, \underset{\sim}{\beta}, \gamma) u \otimes \varepsilon_{\mathrm{f}}, r_{t}(\alpha, \underset{\sim}{\beta}, \gamma) U_{\beta \cup \gamma \gamma} v \otimes \varepsilon_{\mathrm{j}}\right\rangle d \omega,
$$

where $V(\alpha, \underset{\sim}{\beta}, \gamma)$ is the product $V_{1} V_{2} \cdots V_{N}$ in which

$$
V_{k}=\left\{\begin{array}{lll}
\Phi\left(\bar{\phi}^{(i)}\left(\cdot, t_{k}\right)\right) U_{t_{k}}^{i} & \text { if } & t_{k} \in \alpha_{i} \\
\Phi\left(\bar{\lambda}^{(i, j)}\left(\cdot, t_{k}\right)\right) U_{t_{k}}^{i} & \text { if } & t_{k} \in \beta_{i}^{j} \\
\Phi\left(\bar{\psi}^{(j)}\left(\cdot, t_{k}\right)\right) & \text { if } & t_{k} \in \gamma_{j}
\end{array}\right.
$$

and $U$ is defined by $\left(U_{\alpha} k\right)(\omega)=k(\omega \cup \alpha),\left(U_{t}^{i} k\right)(\omega)=k(\omega \cup(\varnothing, \ldots, \varnothing, t, \varnothing, \ldots, \varnothing))$ with $t$ in the $i^{\text {th }}$ place.

By extracting the highest component $s=t_{N}$ from $|\omega|$, taking into account each of the possibilities $s \in \alpha_{i}, \beta_{i}^{j}$ or $\gamma_{j}$, applying the integral-sum identity and differentiating, it may be seen that the family $\mathscr{F}$ satisfies the system of ordinary differential equations

$$
\begin{aligned}
\frac{d F}{d t}(u, v ; \mathbf{f}, \mathbf{g}, t)= & \sum_{i} f_{i}(t) F\left(L_{i}^{*}(t) u, v ; \bar{\phi}^{(i)}(\cdot, t) \mathbf{f}, \mathbf{g} ; t\right) \\
& +\sum_{i, j} \bar{f}_{i} g_{i}(t) F\left(M_{i j}^{*}(t) u, v ; \bar{\lambda}^{(i, j)}(\cdot, t) \mathbf{f}, \mathbf{g} ; t\right) \\
& +\sum_{j} g_{j}(t) F\left(N_{j}^{*}(t) u, v ; \bar{\psi}^{(j)}(\cdot, t) \mathbf{f}, \mathbf{g} ; t\right) \\
& +F\left(K^{*}(t) u, v ; \mathbf{f}, \mathbf{g}, t\right) \\
F(u, v, \mathbf{f}, \mathbf{g}, 0)= & \left\langle u \otimes e_{\mathbf{f}}, v \otimes e_{\mathbf{g}}\right\rangle .
\end{aligned}
$$

On the other hand, applying the commutation relation

$$
U_{s}^{k} \Phi(\phi)=\phi_{k}(s) \Phi(\phi) U_{s}^{k} \quad \text { for } \quad \phi=\operatorname{diag}\left[\phi_{1}, \ldots, \phi_{n}\right]
$$

and the integral-sum identity, it may be seen that

$$
F(u, v ; \mathbf{f}, \mathbf{g} ; t)=\left\langle u \otimes \varepsilon_{\mathbf{f}}, X_{t} v \otimes \varepsilon_{\mathbf{g}}\right\rangle
$$


where $X_{t}$ is given by (6.2). Combining (6.3) and (6.4) we see that $X$ is a solution of (6.1). Uniqueness for solutions of (6.1) follows by minor modifications of the arguments employed in [H P 1].

Remark. Equation (6.2) makes good sense when $k \in \mathscr{K}$, moreover $X_{t} k \in \mathscr{K}$ for each $t$. Here $\mathscr{K}$ has to be redefined to take into account the initial Hilbert space $h_{0}$ :

$$
\mathscr{K}:=\left\{k \in L^{2}\left(\Gamma ; h_{0}\right): a^{N} k \in L^{2}\left(\Gamma ; h_{0}\right) \forall a>0\right\} .
$$

The quantum Itô formula [H P 1] yields sufficient conditions for a process $X$ satisfying (6.1) to be isometry valued and necessary conditions for it to be co-isometry or unitary valued. The following are necessary conditions for $X$ to be unitary valued:

(i) $M_{i j}=U_{i j}-\delta_{i j}$, where $\left\{U_{i j}\right\}$ is unitary $M_{n}\left(\mathscr{B}\left(h_{0}\right)\right)$-valued;

(ii) $N_{j}=\sum_{i} L_{i}^{*} U_{i j}$;

(iii) $K=i H-\frac{1}{2} \sum_{i} L_{i}^{*} L_{i}$, where $H$ is self-adjoint valued;

(iv) $\phi^{(i)}$ and $\psi^{(i)}$ are unitary (diagonal) $M_{n}(\mathbb{C})$-valued;

(v) $\left\{U_{i j} \otimes \Phi\left(\lambda^{i j}\right)+\delta_{i j} \otimes\left[I-\Phi\left(\lambda^{i i}\right)\right]\right\}$ is unitary $M_{n}\left(\mathscr{B}\left(h_{0} \otimes L^{2}\left(\Gamma^{n}\right)\right)\right)$-valued;

(vi) $N_{j} \Phi\left(\psi^{(j)}\right)+L_{j}^{*}\left[\Phi\left(\bar{\phi}^{(j)}\right)-\Phi\left(\bar{\phi}^{(j)} \lambda^{j j}\right)\right]+\sum_{i} L_{i}^{*} U_{i j} \Phi\left(\bar{\phi}^{(i)} \lambda^{i j}\right)=0$;

(vii) $L_{j}^{*} \Phi\left(\bar{\phi}^{(j)}\right)+N_{j}\left[\Phi\left(\psi^{(j)}\right)-\Phi\left(\bar{\lambda}^{j j} \psi^{(j)}\right)\right]+\sum_{i} N_{i} U_{j i}^{*} \Phi\left(\bar{\lambda}^{i i} \psi^{(i)}\right)=0$

they are also sufficient conditions for $X$ to be isometry valued. We leave the non-trivial question of whether (6.5) implies unitarity for the solution of (6.1), and indeed the more general question of whether reasonable conditions may be found on a kernel $x$ for the corresponding operator:

$$
(X k)(\sigma)=\int \sum_{\alpha \cup \beta} \cup \gamma=\sigma(\omega, \alpha, \underset{\sim}{\beta}, \gamma) k(\omega \cup \beta \cdot \cup \gamma) d \omega
$$

to be unitary.

Remark. The following special cases of isometry-valued processes satisfying a quantum stochastic differential equation of the type (6.1) may be noted:

(i) $\psi^{(j)}=\bar{\phi}^{(j)} ; \lambda^{i, j}=\phi^{(i)} \bar{\phi}^{(j)}$;

(ii) $\psi^{(j)}=\bar{\phi}^{(j)} ; \lambda^{i j}=\delta_{i j} I_{n}$ on $\{(s, t): s<t\} ; N_{j}=-L_{j}^{*} U_{j j}$ (i.e. $\left.\sum_{i \neq j} L_{i}^{*} U_{i j}=0\right)$;;

(iii) $\psi^{(j)}=\bar{\phi}^{(j)} ; \lambda^{i j}=0$ on $\{(s, t): s<t\} ; N_{j}=-L_{j}^{*}$;

and when $n=1$ :

(iv) $\psi=\bar{\phi} ; \lambda=\chi_{E}$; for each $t$ either $\lambda_{t} \equiv 1$ or $N(t)=-L^{*}(t)$, where $E \subset \mathbb{R}_{+}^{2}$ is measurable and contains $\{(s, t): s>t\}$;

(v) $\left(X_{t} k\right)(\sigma)=\sum_{\{\beta \subset \sigma: \sigma \times \beta \subset E\}} \prod_{t \in \beta}\left(U_{t}-I\right) k(\sigma)$;

(vi) $\left(W_{t}^{f, \phi} k\right)(\sigma)=\exp \left\{-\frac{1}{2} \int_{0}^{t}|f|^{2}\right\} \sum_{\alpha \in \sigma} \pi_{\phi}(\sigma, \alpha) \int \pi_{\bar{\phi}}(\sigma, \omega) \varepsilon_{f}\left(\alpha_{1}\right) \varepsilon_{\bar{f}}(\omega) k(\omega \cup \alpha) d \omega$; 
in which conditions (i)-(iv) of (6.5) are understood in each case. (iv) is the general solution to (6.5) in the case $n=1$. (v) is the special case of (iv) in which $K=L=N=0$, and is unitary valued by the characterisation of unitary valued martingales in [HLP]. (vi) is the special case of (iv) in which $K=M=0$ and $h_{0}=\mathbb{C}$-these are analogues of Weyl operators and satisfy

$$
\left.\frac{d}{d x}\right|_{x=0} W_{t}^{x f, \phi}=l_{\tilde{\phi}}\left(f \chi_{[0, t]}\right)-l_{\tilde{\phi}}^{\dagger}\left(f \chi_{[0, t]}\right) .
$$

We end with a few remarks on quantum stochastic integrals. The following extensions may be defined:

$$
\begin{aligned}
{\left[A^{*}(F) k\right](\sigma) } & =\sum_{\alpha \in \sigma}[F(\alpha) k](\bar{\alpha}), \\
A(F) k & =\int F(\omega) U_{\omega} k d \omega, \\
\Lambda(F) & =A^{*}(F U),
\end{aligned}
$$

where $\left(U_{\omega} k\right)(\sigma)=k(\sigma \cup \omega),(6.7)$ is a Bochner integral and the following identity for the singular operators $\left\{U_{\omega}\right\}$ is pertinent:

$$
\int\left\|a^{N} U_{\omega} k\right\|^{2} d \omega=\left\|\left(1+a^{2}\right)^{N / 2} k\right\|^{2} \quad(a>0)
$$

from which, in particular, we see that for each $k \in \mathscr{K}, U_{\omega} k \in \mathscr{K}$ for almost all $\omega$. Thus $k \in \mathscr{D}(A(F))$, the domain of the operator $A(F)$, if and only if $\omega \mapsto F(\omega) U_{\omega} k \in$ $L^{1}(\Gamma ; \mathscr{H})\left(\mathscr{H}=h_{0} \otimes L^{2}(\Gamma)\right)$ and, if $F$ is 1 -particle, the condition: $\int\left\|F(s) \varepsilon_{f}\right\|^{2} d s<\infty \forall f$ (from [HP1]) amounts to $\mathscr{E} \subset \mathscr{D}\left(A(F)\right.$ ). Sufficient conditions for $k$ to lie in $\mathscr{D}\left(A^{*}(F)\right.$ ) or $\mathscr{D}\left(\Lambda(F)\right.$ ) (and also for $A^{*}(F) k, \Lambda(F) k$ to belong to $\mathscr{K}$ ) are obtained from the estimate

$$
\left\|a^{N} A^{*}(F) k\right\|^{2} \leqq \int\left\|(a \sqrt{2})^{N+\# \omega} F(\omega) k\right\|^{2} d \omega \quad(a>0) .
$$

Equations (6.9) and (6.10) follow from the integral-sum identity, as does the fact that the definitions $(6.6-6.8)$ do extend those of [HP1] and that

$$
A(F)^{\dagger}=A^{*}\left(F^{*}\right) ; \quad \Lambda(F)^{\dagger}=\Lambda\left(F^{*}\right) .
$$

An advantage of these definitions is that no adaptedness conditions are required, but we shall not pursue this further here.

Acknowledgements. JML wishes to thank his colleagues at the Delhi Centre of the Indian Statistical Institute for warm hospitality and a stimulating research environment during the academic year 1987-88. He also gratefully acknowledges financial support under the Royal Society-Indian National Science Academy exchange agreement.

\section{References}

[AP] Acardi, L., Parthasarathy, K. R.: A martingale characterisation of canonical commutation and anti-commutation relations. J. Funct. Anal. 77, 211-231 (1988)

[ApH] Applebaum, D. B., Hudson, R. L.: Fermion Itô's formula and stochastic evolutions. Commun. Math. Phys. 96, 473-496 (1984)

[Bar] Bargmann, V.: On unitary ray representations of continuous groups. Ann. Math. 59, 1-46(1954) 
[BaW] Bargmann, V., Wigner, E. P.: Group theoretical discussion of relativistic wave equations. Proc. Natl. Acad. Sci. USA 34, 211-223 (1948)

[Gui] Guichardet, A.: Symmetric Hilbert spaces and related topics. Lecture Notes in Mathematics vol. 261. Berlin, Heidelberg, New York: Springer 1972

[HLP] Hudson, R. L., Lindsay, J. M., Parthasarathy, K. R.: Stochastic integral representation of some quantum martingales in Fock space. In: From local times to global geometry, control and physics. Elworthy, K. D. (ed.) Research Notes in Mathematics vol. 150, pp. 121-131. London: Pitman 1986

[HP1] Hudson, R. .L., Parthasarathy, K. R.: Quantum Itô's formula and stochastic evolutions. Commun. Maths. Phys. 93, 301-323 (1984)

[HP2] Hudson, R. L., Parthasarathy, K. R.: Unification of fermion and boson stochastic calculus, Commun. Math. Phys. 104, 457-470 (1986)

[LM1] Lindsay, J. M., Maassen, H.: The stochastic calculus of bose noise, Preprint, 1988

[LM2] Lindsay, J. M., Maassen, H.: An integral kernel approach to noise. In: Quantum probability and applications III (Proceedings, Oberwolfach 1987). Accardi, L., von Waldenfels, W. (eds.) Lecture Notes in Mathematics, pp. 192-208. Berlin, Heidelberg, New York: Springer 1988

[LP] Lindsay, J. M.: Parthasarathy, K. R.: Rigidity of the Poisson convolution. In: 2nd Heidelberg workshop on quantum probability. Accardi, L., von Waldenfels, W. (eds.) Lecture Notes in Mathematics. Berlin, Heidelberg, New York: Springer to appear, 13pp.

[Maa] Maassen, H.: Quantum Markov processes on Fock space described by integral kernels. In: Quantum probability and applications II. Accardi, L., von Waldenfels, W. (eds.) Lecture Notes in Mathematics vol. 1136, pp.361-374. Berlin, Heidelberg, New York: Springer 1985

[Mac] Mackey: G. W., Unitary representations of group extensions. I, Acta. Math. 99, 265-311 (1958)

[Me1] Meyer, P. A.: Eléments de probabilités quantiques I-V: Sém Prob. XX. Lecture Notes in Mathematics, vol. 1204, pp. 186-312. VI-VIII Sém Prob XXI. Lecture Notes in Mathematics, vol. 1247, pp. 34-80. Berlin, Heidelberg, New York: Springer 1986, 1987

[Me2] Meyer, P. A.: Fock space and probability theory. Bielefeld Preprint, 1985

[P] Parthasarathy, K. R.: A unified approach to classical, bosonic and fermionic brownian motion. In: Proc. Lévy Centenary Col., 1987 (Astérisque, Soc. Math. de France)

[Var] Varadarajan, V. S.: Geometry of quantum theory. New York: van Nostrand, 1968, 1970

Communicated by H. Araki

Received May 28, 1988; in revised form November 28, 1988 